\title{
Innate, translation-dependent silencing of an invasive transposon in Arabidopsis
}

Stefan Oberlin ${ }^{1,2}$, Rajendran Rajeswaran ${ }^{1}$, Marieke Trasser,3,4, Verónica BarragánBorrero ${ }^{1,3}$, Michael A. Schon ${ }^{3}$, Alexandra Plotnikova ${ }^{3}$, Lukas Loncsek ${ }^{3}$, Michael D. Nodine $^{3,5}$, Arturo Marí-Ordóñez $z^{1,3^{*}}$ and Olivier Voinnet ${ }^{1^{*}}$

${ }^{1}$ Department of Biology, Swiss Federal Institute of Technology (ETH), Zurich, 8092, Switzerland.

${ }^{2}$ Current address: Department of Microbiology and Immunology, UCSF Diabetes Center, University of California, San Francisco, USA.

${ }^{3}$ Gregor Mendel Institute of Molecular Plant Biology (GMI) of the Austrian Academy of Sciences, Vienna, 1030, Austria.

${ }^{4}$ Vienna BioCenter PhD Program, Doctoral School of the University of Vienna and Medical University of Vienna, Austria.

${ }^{5}$ Laboratory of Molecular Biology, Wageningen University, Wageningen 6708 PB, The Netherlands.

${ }^{*}$ For correspondence:

Olivier Voinnet: voinneto@ethz.ch

Arturo Marí-Ordóñez: arturo.mari-ordonez@gmi.oeaw.ac.at 


\section{Abstract}

2 Co-evolution between hosts' and parasites' genomes shapes diverse pathways of

3 acquired immunity based on silencing small (s)RNAs. In plants, sRNAs cause

4 heterochromatinization, sequence-degeneration and, ultimately, loss-of-autonomy of

5 most transposable elements (TEs). Recognition of newly-invasive plant TEs, by

6 contrast, involves an innate antiviral-like silencing response. To investigate this

7 response's activation, we studied the single-copy element EVADÉ (EVD), one of few

8 representatives of the large Ty1/Copia family able to proliferate in Arabidopsis when

9 epigenetically-reactivated. In Ty1/Copia-elements, a short subgenomic mRNA

10 (shGAG) provides the necessary excess of structural GAG protein over the catalytic

11 components encoded by the full-length genomic fIGAG-POL. We show here that the

12 predominant cytosolic distribution of shGAG strongly favors its translation over mostly-

13 nuclear fIGAG-POL, during which an unusually intense ribosomal stalling event

14 coincides precisely with the starting-point of sRNA production exclusively on shGAG.

15 mRNA breakage occurring at this starting-point yields unconventional 5'OH RNA

16 fragments that evade RNA-quality-control and concomitantly likely stimulate RNA-

17 DEPENDENT-RNA-POLYMERASE-6 (RDR6) to initiate sRNA production. This

18 hitherto-unrecognized "translation-dependent silencing" (TdS) is independent of 19 codon-usage or GC-content and is not observed on TE remnants populating the 20 Arabidopsis genome, consistent with their poor association, if any, with polysomes.

21 We propose that TdS forms a primal defense against de novo invasive TEs that 22 underlies their associated sRNA patterns. 


\section{Introduction}

24 Transposable elements (TEs) colonize and threaten the integrity of virtually all

25 genomes (Huang et al, 2012). Chromosomal rearrangements caused by their highly-

26 repetitive nature (Fedoroff, 2012) are usually circumvented by cytosine methylation

27 and/or histone-tail modifications at their loci-of-origin. The ensuing heterochromatic

28 DNA is not conducive to transcription by RNA Pol II, bringing TEs into an epigenetically

29 silent transcriptional state (Allshire \& Madhani, 2018). This "transcriptional gene

30 silencing" (TGS) is observed at the majority of TE loci in plants, including the model species Arabidopsis thaliana, and causes, over evolutionary times, accumulating mutations resulting in mostly degenerated, non-autonomous entities (Vitte \& Bennetzen, 2006; Civáň et al, 2011). Nonetheless, the genome-invasiveness of these remnants remains evident by their methyl cytosine-marked DNA, which is perpetuated over generations by METHYL-TRANSFERASE 1 (MET1), among other factors. MET1 reproduces symmetrical methylation sites from mother- to daughter-strands during DNA replication (Kankel et al, 2003) aided by the (hetero)chromatin remodeler DEFICIENT IN DNA METHYLATION 1 (DDM1) (Saze et al, 2003; Zemach et al, 2013).

Loss of MET1 or DDM1 functions in Arabidopsis leads to genome-wide demethylation, transcriptional reactivation of many TE remnants, and mobilization of a small portion of intact, autonomous TEs (Mirouze et al, 2009; Tsukahara et al, 2010). Their proliferation together with genome-wide deposition of aberrant epigenetic marks

44 likely explains why met1 and $d d m 1$ mutants accumulate increasingly severe genetic and phenotypic burdens over inbred generations (Vongs et al, 1993). However, such secondary events can be avoided by backcrossing the first homozygous generation of 
ddm1- or met1-derived mutants with wild type plants, upon which continuous selfing of F2 plants creates "epigenetic recombinant inbred lines" (epiRILs). These harbor only mosaics of de-methylated DNA while maintaining wild-type (WT) MET1 and DDM1 functions (Teixeira et al, 2009; Reinders et al, 2009a). One such met1 epiRIL, epi15, endows epigenetic reactivation of the autonomous, long terminal repeat (LTR) retroelement EVADÉ (EVD) in theTy1/Copia family, which is one of the most proliferative families in plants (Vitte \& Panaud, 2005). Of the two EVD copies in the Arabidopsis Col-0 genome, only one is reactivated in epi15 (Marí Ordóñez et al, 2013).

By providing a proxy for a de novo genomic invasion, this reactivation granted a unique opportunity to grasp how, over multiple inbred generations, newly invasive TEs might be detected and eventually epigenetically silenced (Marí Ordóñez et al, 2013). (PTGS) akin to that mounted against plant viruses (Voinnet, 2005; Marí Ordóñez et al,

2013). Antiviral RNA-DEPENDENT RNA POLYMERASE 6 (RDR6) produces cytosolic, long double-stranded (ds)RNAs from EVD-derived transcripts, which are then processed by DCL4 or DCL2, two of the four Arabidopsis Dicer-like RNase-III enzymes, into populations of respectively 21- and 22-nt small interfering (si)RNAs. However, despite their loading into the antiviral PTGS effectors ARGONAUTE1 and ARGONAUTE2 (AGO1/2), they do not suppress expression of EVD's increasingly more abundant genomic copies. This ultimately gives way to DCL3, instead of DCL4/2,

67 to process the RDR6-made long dsRNAs into 24-nt siRNAs. In association with AGO4clade AGOs, these species guide RNA-directed DNA methylation (RdDM) of EVD copies. Initially localized within the EVD gene body, it later spreads into the LTRs to eventually shut down the expression of EVD genome-wide via TGS (Marí Ordóñez et al, 2013). 
A key, unsolved question prompted by this proposed suite of events pertains to

73 the mechanisms whereby RDR6 is initially recruited onto EVD, and more generally on

74 newly invasive TEs, during the primary antiviral-like silencing phase. "Homology-" or

75 “identity"-based silencing entails sequence complementarity between TE transcripts

76 and host-derived small RNAs. Loaded into AGOs, they likely attract RDR6

77 concomitantly to silencing execution. One such type of PTGS occurs with TEs

reactivated in ddm1/met1 mutants, which, by displaying complementarity mostly to

host-encoded microRNAs, spawn "epigenetically-activated siRNAs" (easiRNAs) in an

AG01-dependent manner (Creasey et al, 2014). easiRNA production likely entails

substantial co-evolution between host and TE genomes (Sarazin \& Voinnet, 2014)

because miRNAs usually target short and highly conserved TE regions, including the

(Šurbanovski et al, 2016; Borges et al, 2018). Another form of acquired immunity underlying identity-based silencing is conferred by siRNAs derived from relics of previous genome invasions by the same or sequence-related TE(s) (Fultz \& Slotkin, 2017).

New intruder TEs are unlikely to engage either form of identity-based silencing, as indeed noted for EVD (Creasey et al, 2014). Thus, RDR6-dependent PTGS initiation should involve intrinsic features of the TEs themselves (Sarazin \& Voinnet, 2014). In the yeast Cryptococcus neoformans, stalled spliceosomes on suboptimal TE

92 introns provide an opportunity for an RDR-containing complex to co-transcriptionally 93 initiate such innate PTGS (Dumesic et al, 2013). Studies of transgene silencing in 94 plants (Luo \& Chen, 2007; Thran et al, 2012) have advocated other possible 95 mechanisms, though none has yet been linked to epigenetically reactivated TEs. 96 These studies describe how uncapped, prematurely terminated or non-polyadenylated 
transcripts might stimulate RDR activities when they evade or overwhelm RNA quality control (RQC) pathways that normally degrade these "aberrant" RNAs (Parent et al, translation-coupled RNA degradation as a consequence of suboptimal codon usage and low GC content might trigger RDR-dependent silencing in plants (Kim et al, 2021). Initiation of innate PTGS in the context of EVD likely ties in with an unusual process of splicing-coupled premature cleavage and poly-adenylation (PCPA) shared genomes (Oberlin et al, 2017).On the one hand, an unspliced and full-length (fI) GAG-

$106 P O L$ isoform codes for a polyprotein processed into protease, integrase/reverse107 transcriptase-RNase and GAG nucleocapsid components. On the other hand, a spliced and prematurely terminated short (sh) GAG subgenomic isoform is solely dedicated to GAG production. Though less abundant than the fIGAG-POL mRNA, shGAG is substantially more translated (Oberlin et al, 2017). This presumably results

111 in a molar excess of structural GAG for viral-like particle (VLP) formation compared to

112 Pr-IN-RT-RNase required for reverse-transcription (RT) and, ultimately, mobilization 113 (Oberlin et al, 2017; Lee et al, 2020). Supporting the notion that genome expression 114 of Ty1/Copia elements influences PTGS initiation, EVD-derived RDR6-dependent 115 siRNAs do not map onto the unspliced fIGAG-POL mRNA, but instead specifically onto 116 the spliced shGAG transcript of which, intriguingly, they only cover approximately the 117 3' half (Oberlin et al, 2017).

118 Here, we show that differential subcellular distribution of the two mRNA 119 isoforms due to splicing-coupled PCPA accounts for the peculiar EVD siRNA 120 distribution and activity patterns. While the fIGAG-POL isoform remains largely 121 nuclear, the shGAG mRNA is enriched in the cytosol and endows vastly 
122 disproportionate translation of shGAG over fIGAG-POL. However, a previously

123 uncharacterized innate PTGS process accompanies active shGAG translation,

124 manifested as a discrete and unusually intense ribosome stalling event independent

125 of codon usage or GC content, among other tested parameters. Ribosome stalling

126 coincides precisely with the starting point of shGAG siRNA production and maps to

127 the 5 ' ends of discrete, shGAG-derived RNA breakage fragments. These harbor

128 unconventional $5^{\prime} \mathrm{OH}$ termini that prevent their RQC-based degradation via 5'P-

129 dependent XRN4 action (Stevens, 2001; Peach et al, 2015). Based on the well-

130 documented substrate competition between XRN4 and RDR6(Gazzani, 2004; Gy et

131 al, 2007b; Gregory et al, 2008; Moreno et al, 2013; Martínez de Alba et al, 2015), we

132 suggest that the $5{ }^{\prime} \mathrm{OH}$ status of breakage fragments licenses their conversion into

133 dsRNA by RDR6, thereby initiating PTGS of EVD. We further show that splicing-

134 coupled PCPA suffices to recapitulate this "translation-dependent silencing" (TdS) in

135 reporter-gene settings. Given that Ty1/Copia retroelements share a PCPA-based

136 genome expression strategy (Oberlin et al, 2017), we contend that TdS forms a

137 generic and primal defense against de novo invasive TEs that shapes the siRNA 138 patterns initially associated with them. 


\section{shGAG is the main source and target of EVD-derived siRNAs}

142 Arabidopsis lines constitutively overexpressing an LTR-deficient but otherwise intact

143 form of EVD driven by the 35S promoter (35S:EVDwt) recapitulate the restriction of

144 EVD siRNA to the 3' part of the shGAG sequence(Marí Ordóñez et al, 2013; Oberlin

145 et al, 2017) (Fig.1A-B). We explored EVD transcripts levels in 35S:EVD $D_{w t}$ in WT

146 (siRNA-proficient) as opposed to rdr6 (siRNA-deficient) background (Fig.1B, S1A).

147 Both in RNA blot and qRT-PCR analyses, the spliced shGAG mRNA levels were

148 increased in $r d r 6$ compared to WT, whereas those of unspliced fIGAG-POL were 149 globally unchanged (Fig.1C-D). Accordingly, accumulation of the GAG protein 150 mainly produced via shGAG translation (Oberlin et al, 2017) - was higher in rdr6 compared to WT background (Fig.1E). Essentially identical results were obtained upon epigenetic reactivation of endogenous EVD in non-transgenic Arabidopsis with the $d d m 1$ single- versus ddm1 rdr6 double mutant background (Fig.S1B-E). Following EVD mobilization from an early (F8) to a more advanced (F11) epi15 inbred generation (Marí Ordóñez et al, 2013) revealed that its progressively increased copy number correlates with progressively higher steady-state levels of EVD-derived transcripts and

157 EVD-derived siRNAs (Fig.S1F-G). Again, these siRNAs disproportionately target the shGAG relative to fIGAG-POL mRNA from F8 to F11 (Fig.S1H). Collectively, these results indicate that PTGS activated de novo by EVD is both triggered by, and targeted 160 against, the spliced shGAG mRNA. Therefore, features associated with shGAG, but 161 not fIGAG-POL, likely stimulate RDR6 recruitment, which we explored by testing current models for PTGS initiation from TEs and transgenes. 
165 Though unlikely (Creasey et al, 2014; Sarazin \& Voinnet, 2014), we first considered 166 that production of RDR6-dependent siRNAs from shGAG might require its cleavage 167 by miRNAs via the easiRNA pathway (Creasey et al, 2014). Arabidopsis miRNA 168 biogenesis depends on DCL1 and the dsRNA-binding protein HYL1, among other 169 factors (Brodersen \& Voinnet, 2006). Analyses of publicly available sRNA-seq data 170 (Creasey et al, 2014) showed, however, that epigenetically reactivated EVD spawns 171 qualitatively and quantitatively identical shGAG-only siRNAs in both ddm1 single and $172 d d m 1 d c / 1$ double mutants (Fig.1F-G). Moreover, levels of shGAG siRNA, shGAG 173 mRNA, and GAG protein remained unchanged in 35S:EVD $D_{w t}$ plants with either the 174 WT, hypomorphic dc/1-11 or loss-of-function hyl1-2 background (Fig.S2A-D). By contrast and as expected, production of trans-acting (ta)siRNAs, which is both miRNAand RDR6-dependent, was dramatically reduced and the levels of tasiRNA precursors

177 and target transcripts enhanced in both mutant backgrounds (Fig.S2). Therefore, 178 RDR6 recruitment to the spliced shGAG mRNA is unlikely to involve endogenous 179 miRNAs via an identity-based mechanism. We then explored known innate processes 180 of PTGS initiation instead. EVD-like siRNA accumulation and activity patterns

184 Some cases of transgene-induced PTGS correlate with a lack of polyadenylation due 185 to aberrant RNA transcription (Luo and Chen 2007). We ruled that this feature underlies EVD-derived siRNA production because shGAG displays no overt 
187 polyadenylation defects regardless of the onset of PTGS (Fig.S3). Next, we

188 considered splicing defects, such as inaccurate splicing or spliceosome stalling, and 189 premature transcriptional termination as possible PTGS triggers, two processes 190 previously independently linked to innate, RDR-dependent siRNA production in plants 191 and fungi ((Dalakouras et al, 2019; Dumesic et al, 2013). Ty1/Copia elements have 192 introns that are significantly longer than those of Arabidopsis genes. Moreover, 193 shGAG undergoes atypical splicing-coupled PCPA (Oberlin et al, 2017). When 194 engineered between the GFP and GUS sequences of a translational fusion, the 195 shGAG intron and proximal PCPA signal spawn unspliced fIGFP-GUS and spliced

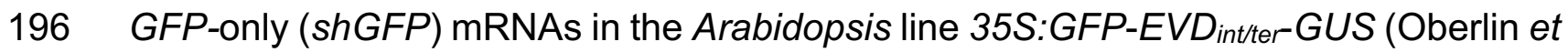
197 al, 2017) (Fig.2A-B;Fig.S4A). Since this artificial system recapitulates the production 198 of respectively fIGAG-POL and shGAG, we asked if an EVD-like siRNA pattern was 199 likewise reproduced.

200 The majority of RDR6-dependent 21-nt siRNAs mapped to the GFP, but not the 201 GUS region downstream of the PCPA signal (Fig.2C-D) suggesting that, just like 202 shGAG in EVD, the spliced shGFP mRNA is the main source of siRNAs in GFP-

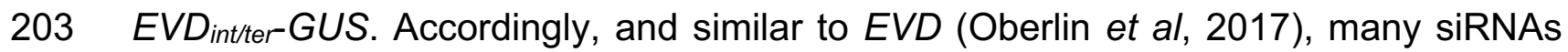
204

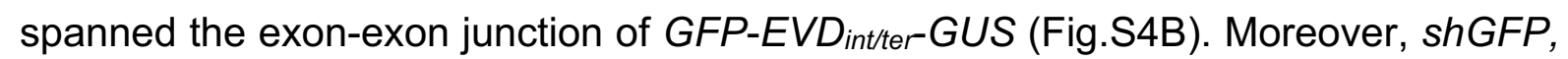
205 unlike fIGFP-GUS, over-accumulated in GFP-EVDint/ter-GUS plants with the rdr6 206 background (Fig.2B, Fig.S4A), indicating that only shGFP is efficiently targeted by 207 PTGS (Fig.2B). Therefore, in the reconstituted setting, the intron and PCPA signal 208 found in shGAG suffice to spawn RDR6-dependent siRNAs displaying accumulation 209 and activity patterns resembling those generated in the authentic EVD context (Fig.1A210 D). 


\section{Neither splicing nor intron-retention per se initiate RDR6 recruitment}

213 The above result prompted us to investigate a potential facilitating role for splicing in

214 shGAG siRNA biogenesis or, conversely, a role for intron-retention in inhibiting RDR6

215 recruitment to fIGAG-POL. We used previously engineered Arabidopsis EVD-

216 overexpression lines with a point-mutated U1 snRNP-binding site (35S:EVD $\left.D_{m 1}\right)$ or a

217 fully deleted intron (35S:EVD $D_{\Delta i}$ ) (Oberlin et al, 2017) (Fig.3A-C). 35S:EVD ${ }_{\Delta i}$ spawns

218 fully matured shGAG transcripts that do not associate with the spliceosome, leading

219 exclusively to prematurely terminated and polyadenylated mRNA species with a stop

220 codon (Oberlin et al, 2017) (Fig.3B, 3D, 3E, Fig.S5A). However, lack of the intron, and

221 hence splicing, did not prevent RDR6-dependent siRNA production from 35S:EVD ${ }_{\Delta i}$,

222 which was comparable to that of 35S:EVD $D_{w t}$ (Fig.3E). Moreover, the shGAG mRNA and GAG protein levels from 35S:EVD $D_{\Delta i}$ were higher in an rdr6 compared to WT background (Fig.3D-F), indicating that EVD's unconventional splicing is unlikely to underpin shGAG siRNA production.

To test the alternative possibility that intron-retention or specific sequences within

227 the EVD intron prevent siRNA biogenesis from fIGAG-POL, we analyzed the siRNAs 228 from 35S:EVD $D_{m U 1}$. Impeding $\mathrm{U} 1$ binding and its inhibitory action on PCPA causes a complete lack of splicing in $E V D_{m U 1}$ (Fig.3C-D). This generates short unspliced

230 transcripts, alternatively terminated at the cognate shGAG terminator or at an intronic 231 cryptic site previously mapped by 3' RACE (Oberlin et al, 2017), both detected here 232 by northern analysis (Fig.3C-E, Fig.S5A). Both alternatively terminated transcripts 233 likely undergo translation, albeit largely unproductively (Fig.3F), because low levels of 234 cryptic GAG translation products were detectable in rdr6 compared to WT (Fig.S5B). $235 E V D_{m U 1}$ bestowed RDR6-dependent siRNA production expanding - as expected from 236 its non-spliceable nature - into the retained intron sequence (Fig.3E). The near- 
complete lack of siRNAs downstream of the intron (Fig.S5A), by contrast, suggested

238 that both cryptically terminated shGAG transcripts are mainly involved in recruiting

239 RDR6. Therefore, even though the shGAG intron and PCPA signal suffice to trigger

240 PTGS from EVD and GFP-EVD ${ }_{\text {int/ter }}$ GUS (Figs.1-2), neither splicing nor intron-

241 retention per se seem to initiate PTGS. This suggests that splicing-coupled PCPA

242 does not co-transcriptionally condition the sensitivity of shGAG to RDR6 but, rather,

243 downstream in the gene expression pathway.

244

245 RDR6 recruitment onto shGAG likely requires translation

246 Splicing-coupled PCPA, conserved among Arabidopsis Ty1/Copia elements,

247 correlates with the over-representation of shGAG on polysomes as opposed to the 248 paradoxically more abundant fIGAG-POL (Oberlin et al, 2017). However, among the

$249 d d m 1$ - or met1- reactivated Ty1/Copia elements sharing the same genome expression 250 strategy, only EVD spawns detectable RDR6-dependent shGAG siRNAs (Oberlin et 251 al, 2017), prompting us to explore the basis for this difference. Polysome association, 252 independently of translation efficiency, is the most decisive prerequisite for any given 253 RNA to engage the translation machinery. For instance, many non-coding RNAs are mostly nuclear (Khanduja et al, 2016), and aberrant (e.g. uncapped and/or poly(A)-) mRNAs are actively degraded by RQC, both of which explain their general absence 256 from polysomes (Doma \& Parker, 2007). We conducted genome-wide correlation 257 analyses between steady-state transcript accumulation, polysome association, and siRNA levels of reactivated TEs in the $d d m 1$ versus $d d m 1$ rdr6 background by calculating the ratio of polysome-associated versus total mRNA levels. The same approach was applied to Arabidopsis protein-coding compared to non-coding RNAs used as references (Oberlin et al, 2017). This analysis revealed two distinct TE 
populations according to the levels of associated RDR6-dependent siRNAs. On the one hand, approximately $3 / 4$ of $d d m 1$ de-repressed TEs (530/674) display varying degrees of polysome association, some within the range of protein-coding genes (Fig. 4A, quartiles 1-3). However, RDR6-dependent siRNA production does not accompany their reactivation presumably because of their low expression levels (Fig.4B, quartiles 1-3). The remaining $1 / 4(144 / 674)$ of TEs spawn RDR6-dependent siRNAs, correlating with higher RNA expression levels (Fig.4A-B quartile 4). Nonetheless, unlike those of quartiles 1-3, these TEs, almost exclusively composed of degenerated LTR/Gypsy elements (i.e. elements shorter than-full-length reference ORFs; Fig.4C), resemble non-coding RNAs in being poorly associated with polysomes, if at all (Fig. 4A, quartile

272 4). By contrast, EVD is the sole LTR/Copia element within quartile 4, in which it is one 273 of the most strongly polysome-associated elements that concurrently spawn 21-22-nt siRNAs. Furthermore, when the two EVD isoforms are considered separately, shGAG emerges as a clear outlier by being associated with polysomes to the same extent as 276 protein-coding mRNAs, (Fig.4A, quartile 4, inlay). fIGAG-POL, by contrast, displays 277 low polysome association albeit higher than most degenerated LTR/Gypsy elements 278 populating quartile 4. In summary, shGAG, compared to fIGAG-POL, is both vastly overrepresented on polysomes (Oberlin et al, 2017) and is the major, if not unique source of EVD-derived siRNAs (Fig.1, S1, Fig.3). This analysis suggests, therefore, that translation is the step stimulated by splicing-coupled PCPA of shGAG, upon which RDR6 is recruited specifically onto this mRNA isoform. 
286 To test if differential translation due to splicing-coupled PCPA indeed underlies siRNA 287 production from shGAG as opposed to fIGAG-POL, we used GFP-EVDint/ter-GUS, from 288 which the two EVD RNA isoforms and associated siRNA production/activity patterns 289 are recapitulated (Fig.2). Of the shGAG-like shGFP- and fIGAG-POL-like fIGFP-GUS290 mRNAs, only the former produced a detectable protein under the form of free GFP 291 (Fig.S4C-D) despite accumulation of both mRNAs (Fig.2A, Fig.S4A). Free GFP levels 292 were increased in the rdr6 background (Fig.S4C), coinciding with increased shGFP. 293 but unchanged fIGFP-GUS- mRNA levels (Fig.2B). The lack of detectable GFP-GUS 294 fusion protein - the expected product of fIGFP-GUS- in either WT or rdr6 backgrounds 295 (Fig.2D, Fig.S4C-D) was not due to intrinsically poor translatability. Indeed, GFP-GUS 296 was the sole protein detected in independent lines undergoing RDR6-dependent 297 PTGS of 35S:GFP-GUS, a construct identical to 35S:GFP-EVD int/ter-GUS, save the shGAG intron and PCPA signal (Fig.5A-B). As expected, the GFP-GUS fusion protein and GFP-GUS mRNA levels were strongly enhanced in the rdr6 versus WT 300 background (Fig.5A-B). Yet, in contrast to GFP-EVD ${ }_{\text {int/ter }}$ GUS, from which siRNAs are restricted to shGFP, the siRNAs from GFP-GUS encompassed both the GFP and GUS sequences (Fig.5A). These results therefore indicate that splicing-coupled PCPA promotes selective translation of, and PTGS initiation from, shGAG-like as opposed to $f I G A G-P O L$-like mRNA isoforms.

\section{Intron retention causes selective nuclear seclusion of fIGAG-POL-like mRNAs}

307 What mechanism linked to splicing-coupled PCPA might underpin the differential 308 translation of shGAG-like versus fIGAG-POL-like mRNAs? Noteworthy, splicing 309 generally enhances mRNA nuclear export and translation (Valencia et al, 2008; 310 Sørensen et al, 2017). Conversely, polyadenylated, unspliced mRNAs are retained in 
311 the nucleus in Arabidopsis and only exported to the cytoplasm upon splicing (Jia et al,

312 2020). Moreover, 5' splice motifs and U1 snRNP binding promote chromatin tethering

313 of long non-coding RNAs in animal cells (Lee et al, 2015; Yin et al, 2020). We thus

314 tested if intron-retention might promote nuclear sequestration of the unspliced fIGFP.

315 GUS and fIGAG-POL or if, conversely, splicing might favor export of shGFP and 316 shGAG to the cytoplasm, thereby selectively promoting their translation. We 317 performed nucleo-cytosolic fractionation (Fig.S5C) to analyze the relative distributions 318 of EVD-derived RNA isoforms produced in 3S:EVD $D_{w t}$ or $35 S: G F P-E V D_{\text {int/ter }}$ GUS 319 plants, using spliced/unspliced isoform-specific PCR amplification. Additionally, 320 unspliced isoforms were selectively analyzed using qPCR primer sets designed to 321 amplify sequences located near the 3' end of fIGAG-POL or fIGFP-GUS, and absent 322 from shGAG and shGFP (Fig.1A, Fig.2A). A similar approach was used to differentiate 323 the unspliced versus spliced ACTIN mRNA (Fig.S5D). Finally, the nuclear-only 324 snoRNA U5 (Fig.5C) was used as a control to assess the quality of nuclear 325 enrichments. To optimize accumulation of both types of RNA isoforms, the experiments were all conducted in the PTGS-deficient rdr6 background. The analysis revealed strikingly distinct nucleo-cytosolic distribution patterns for 328 the full-length versus short spliced mRNAs from both systems. Indeed, while the 329 spliced shGFP and shGAG were found predominantly in the cytosol (Fig.5C), fIGAG$330 P O L$ and fIGFP-GUS were strongly enriched in nuclear fractions (Fig.5C, Fig.S5D). To 331 validate that nuclear unspliced full-length transcripts are bona fide poly $(\mathrm{A})^{+} \mathrm{mRNAs}$ as opposed to nascent transcripts or splicing intermediates, cDNA from the same RNA samples was synthesized using exclusively oligo-dT to capture polyadenylated RNAs 334 only. This approach generated comparable results (Fig.5D), indicating that nuclear 335 full-length transcripts are properly terminated mRNAs. Corresponding results were 
obtained in epi15 F11 plants displaying endogenous EVD reactivation (Fig.S5E-F).

337 Collectively, these findings suggest that the unique splicing behavior of EVD - which

338 is recapitulated in GFP-EVD $D_{\text {int/ter }}$ GUS - not only allows production of the GAG-

339 encoding shGAG subgenomic mRNA, but simultaneously promotes nuclear retention

340 of $f / G A G-P O L$. This is likely contributing to the disproportionate translation of shGAG

341 over fIGAG-POL, although we do not exclude the involvement of other processes.

342 Under these premises, splicing-coupled PCPA likely predisposes shGAG, as opposed

343 to fIGAG-POL, to one or several co-translational processes which, in turn, signal(s)

344 RDR6 recruitment.

346 Saturation of co-translational mRNA decay unlikely triggers shGAG siRNA 347 production

348 In plants and fungi, decapping coupled to 5'->3' exonucleolytic activity operated by 349 cytosolic XRN proteins regulate the intrinsic half-life of most actively translated 350 transcripts by degrading decapped mRNAs after the last translating ribosome 351 (Kastenmayer \& Green, 2000; Hu et al, 2009; Pelechano et al, 2015; Yu et al, 2016). 352 Of the three Arabidopsis XRNs, XRN2 and XRN3 are nuclear, whereas XRN4 is 353 cytosolic and, hence, mediates co-translational mRNA decay (Gregory et al, 2008; 354 Kurihara, 2017; Yu et al, 2016). Remarkably, transcripts undergoing improper decapping and/or XRN4-mediated exonucleolysis constitute competing substrates for RDR6 in Arabidopsis (Gazzani, 2004; Gy et al, 2007a; Gregory et al, 2008; Moreno et al, 2013; Martínez de Alba et al, 2015) (Fig.6A). For instance, loss-of-RDR6 function suppresses the lethality of decapping mutants by preventing production of undesirable 359 siRNAs from hundreds of endogenous mRNAs (Martínez de Alba et al, 2015). 360 Conversely, loss of XRN4 activity enhances RDR6-dependent PTGS (Gy et al, 2007a; 
361 Gregory et al, 2008; Moreno et al, 2013). These observations strongly suggest that

362 RDR6-dependent PTGS takes over co-translational mRNA decay when this process

363 becomes saturated by highly abundant and/or highly translated mRNAs.

co-translational decay of shGAG and thereby concurrently promote RDR6 action

(Fig.6A). This would predict an accumulation of RNA degradation fragments (reflecting

XRN4 activity) coinciding with siRNA accumulation. PARE (parallel amplification of

RNA ends) and related methods map mostly XRN4 products associated with co-

translational decay as well as non-translational RNA cleavage events, e.g. miRNA-

mediated slicing of non-coding RNAs (Gregory et al, 2008; Schon et al, 2018). We

371 therefore conducted nanoPARE analyses, which capture both capped and uncapped

372 RNA fragments (Schon et al, 2018), in ddm1 vs WT Arabidopsis (Fig.S6A).

373 Simultaneously, mRNA-seq (i.e. SMART-seq2) was conducted on the same RNA to

374 monitor gene expression (Schon et al, 2018). Analysis of TAS1c, which undergoes

375 miR173-mediated slicing, confirmed that the ensuing 3' RNA cleavage fragment, a

376 common substrate of XRN4 (Schon et al, 2018), was readily detected in both

377 backgrounds, despite spawning vast amounts of RDR6-dependent siRNAs (Fig.6B).

378 Analyzing EVD upon its reactivation in $d d m 1$ revealed a low level of RNA degradation

379 fragments spanning the entirety of EVD despite the siRNAs being exclusively derived

380 from shGAG. Had RNA degradation contributed to siRNA biogenesis, these species

381 would be expected to be distributed along the entirety of $E V D$, encompassing both

382 shGAG and fIGAG-POL. Inspection of the housekeeping ACT2 locus revealed a

383 similar ORF-spanning degradation pattern, albeit at substantially higher levels ( 10-

384 folds), presumably reflecting the higher transcript abundance. However, ACT2 does

385 not spawn siRNAs (Fig. 6B). These observations therefore reveal no overt correlation 
between abundance of RNA degradation products, siRNA production and/or polysome association.

The above results did not formally exclude the possibility that at least some EVDassociated degradation products identified by nanoPARE might contribute to siRNA biogenesis via competing RDR6 vs XRN4 activities. This would be genetically

loss in rdr6 (Gy et al, 2007a; Gregory et al, 2008). To test this idea without the potential complication of EVD overexpression artificially saturating XRN4 activity in 35S:EVD $D_{w t}$, we introgressed the xrn4 null-mutation into epi15 at the early F8 inbred generation,

contributing to co-translational mRNA decay, should not influence siRNA production.

398 Finally, the rdr6 mutation was introgressed in parallel, to prevent shGAG siRNA 399 biogenesis. We analyzed two-to-three independent lineages with WT versus 400 homozygous mutant backgrounds isolated from segregating F2s. However, neither $x r n 4$ nor $x r n 2 / x r n 3$ differed from the WT background with regard to EVD expression, 402 copy number, or shGAG siRNA levels (Fig.6C-E, Fig.S6B-G). In contrast, EVD 403 expression and copy numbers were increased in rdr6, coinciding with reduced shGAG 404 siRNA levels (Fig.6F-H). We conclude from these collective results that saturation of XRN4-dependent co-translational mRNA decay (Fig.6A) is unlikely to underlie shGAG siRNA production. siRNAs are, instead, abruptly spawned from the middle up to the 3' end of shGAG, as if their production coincided with a discrete co-translational event 408 (Fig.6A). A similar rationale should apply to the discrete shGFP-centric siRNA pattern

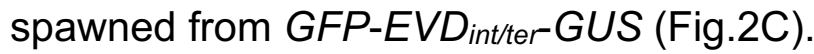


411 The initiation of RDR6 activity coincides with isolated and intense ribosome

412 stalling events

413 To overcome the caveat of EVD cell-specific expression (Marí Ordóñez et al, 2013)

414 and simultaneously investigate which co-translational event(s) might trigger the siRNA

415 patterns in both shGAG and shGFP, we generated RIBO-seq datasets (Ingolia et al,

416 2009) from 35S:EVD ${ }_{w t}$ and 35S:GFP-EVD int/ter-GUS. This resulted in high-quality

417 ribosome footprints (RFPs) displaying the characteristic triplet periodicity (Fig.S7). We

418 found that EVD RFPs in the $35 S$ :EVD $D_{w t}$ background map near-exclusively onto

419 shGAG, underscoring its preferential translation (Fig.4A, 7A). However, a strong and

420 isolated footprint peak was detected near the middle of the shGAG ORF (Fig.7A),

421 suggesting intense ribosome stalling at this position. This stalling peak was also found

422 within the shGAG coding sequence of endogenous EVD in one public ddm1 RIBO-

423 seq library (Kim et al, 2021) (Fig.S8A). By specifying codon occupancy of ribosome P-

424 sites - the sites of peptidyl transfer activity - reflecting the codon dwell time, we found

425 that $>35 \%$ of shGAG translating ribosomes are located on two consecutive codons

426 (pos.148-149) coinciding with this peak (Fig.7B). Having normalized these proportions

427 to ORF lengths, we compared them to those of actively translated Arabidopsis

428 mRNAs. To exclude artefacts from transcripts with low coverage, we restricted our

429 analysis to the most abundant mRNA isoforms with coverage available for more than

$43070 \%$ of ORFs, as described (Sabi \& Tuller, 2015). We found that shGAG ranks among

431 the top 4.01 and $2.77 \%$ (in WT and rdr6 backgrounds respectively) of Arabidopsis

432 transcripts displaying the most intense stalling events (Fig.7C). Remarkably,

433 overlaying siRNAs and codon coverage intensity revealed that the intense stalling

434 position coincides nearly exactly with the 5' starting point of the RDR6-dependent EVD 
siRNA pattern (Fig.7D). Stalling is likely causal, not a consequence of RDR6

436 recruitment, because it also occurs in the rdr6 background (Fig.7A).

To explore further a possible link between discrete, intense ribosome stalling and

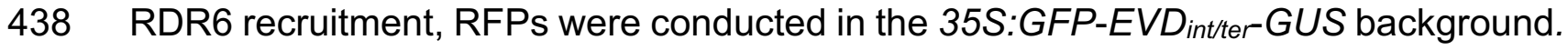

439 As seen above for shGAG versus fIGAG-POL in the EVD context, the analysis 440 confirmed the vastly disproportional translation of shGFP versus fIGFP-GUS 441 (Fig.S8B). It also identified a major stalling site only in the shGFP ORF (whose

442 detection was enhanced in the rdr6 background) in which two prominently covered 443 and consecutive codons (pos. 235-236) accounted for $\sim 40 \%$ of footprints (Fig.S8C).

444 Similarly to shGAG, shGFP ranked among the top 3.21 to $4.14 \%$ Arabidopsis 445 transcripts displaying the most intense stalling events (Fig.S8D). Furthermore, this 446 stalling site was located between major peaks of shGFP siRNAs, in this case, in both

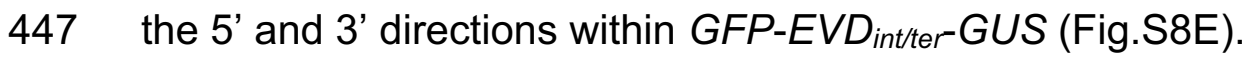

A recent model advocates a possible link between suboptimal codon usage and

449 PTGS initiation in plants (Kim et al, 2021). However, overlaying the Arabidopsis codon 450 adaptation index with the codon coverage of ribosomes on shGAG and shGFP did not 451 reveal any overt correlation between ribosome stalling and codon suboptimality 452 (Fig.S9). The cited study showed that codon optimization in a region corresponding, 453 surprisingly, to the shGAG 3'UTR enhanced translation of a linked luciferase ORF 454 (Kim et al, 2021). Yet, our analysis shows that neither CG nor CG3 content (CG 455 content on the $3^{\text {rd }}$ codon position) overtly influences ribosome association along shGAG or shGFP, let alone the intense stalling event detected on either mRNA 457 (Fig.S10). Two consecutive codons at the stalling site identified on shGAG code for 458 proline and glycine (Fig.S9A) and, interestingly, single prolines and/or glycines at P459 sites correlate with ribosome stalling in animals and fungi (Artieri \& Fraser, 2014; Sabi 
460 \& Tuller, 2015; Zhao et al, 2021). By contrast, the $>45 \%$ codon occupancy on shGFP

461 occurs on unrelated, consecutive glutamate and leucine codons (Fig.S9B). In addition

462 to the identity of some codons, secondary RNA structures have been correlated with

463 ribosome stalling (Doma \& Parker, 2006; Yan et al, 2015; Bao et al, 2020), including

464 G-quadruplexes (Song et al, 2016; Fay et al, 2017). In particular, sites of "ribothrypsis"

465 - a ribosome stalling-induced process recently described in metazoans - are

466 positively correlated with such occurrences within ORFs (Ibrahim et al, 2018). By

467 forming secondary structures, G-quadruplexes are thought to act as "roadblocks"

468 hampering proper ribosome progression during elongation (Song et al, 2016). While

469 G-quadruplex scoring along the shGAG and shGFP mRNA did reveal potential hot

470 spots of such motifs, they were localized far upstream or downstream of each

471 identified ribosome stalling site (Fig.S9). Overall, EVD and GFP-EVD $D_{\text {int/ter-GUS }}$

472 transcripts display similar behavior, whereby RDR6-dependent production of shGAG-

473 or shGFP-only siRNAs coincides with highly localized and unusually intense ribosome

474 stalling events. While stalling events have likely distinct causes for each transcript,

475 they nonetheless appear to stimulate co-translational processing of RNA

476 intermediates that, in turn, serve as RDR6 substrates.

477

478 Ribosome stalling correlates with production of 5'-hydroxy 3'-cleavage

479 fragments that possibly serve as RDR6 substrates

480 As described above, nanoPARE in $d d m 1$ did not reveal any discrete RNA products

481 with 5' ends mapping consistently at, or near, the stalling site in shGAG. We also failed

482 to detect such products using classic 5' RACE (Llave et al, 2002). Noteworthy, this

483 technique relies on a 5' monophosphate (5'P) for RNA ligation of 5' adaptors (Silber 
et al, 1972; Wang \& Fang, 2015). Intriguingly, 5'P was reported to be absent from various 3' cleavage RNA fragments produced co-translationally in budding yeast, including upon ribosome stalling (Peach et al, 2015; Navickas et al, 2020). A lack of $5^{\prime} \mathrm{P}$ is also strongly suspected for the 3' cleavage products of ribothrypsis (Ibrahim et al, 2018). Since siRNA production from EVD initiates just downstream of the major stalling site (codons 148-149), we thus considered the possibility that discrete associated with the above-mentioned processes (Peach et al, 2015; Navickas et al, 2020; Ibrahim et al, 2018; D'Orazio et al, 2019) - might constitute RDR6 templates (Fig.7E).

To explore such a connection and simultaneously characterize and map the 495 ' ends of putative shGAG 3' cleavage fragments, we used the RtcB RNA ligase. RtcB contributes to tRNAs splicing by ligating RNAs with 3'P ends (or 2',3'-cyclic phosphate) to $5^{\prime} \mathrm{OH}$ ends, and was used previously to map co-translational RNA cleavage 498 fragments in yeast (Desai \& Raines, 2012; Peach et al, 2015). A 5' RNA adaptor with 499 a 3'P end was therefore RtcB-ligated to total RNA extracted from plants expressing 500 35S:EVD or non-transgenic controls, both in the rdr6 background. Use of rdr6 501 prevented conversion of potential RDR6 templates into dsRNA as well as the 502 accumulation of confounding cleavage fragments potentially caused by the ensuing 503 secondary siRNAs. The ligated RNA was then subjected to reverse transcription using 504 EVD-specific primers surrounding the major stalling site (Fig. 7F; Region \#1), amplified 505 through PCR, and cloned following standard RACE procedures. Based on the EVD 506 ribosome footprint profile (Fig. 7B), we also investigated two additional regions more 507 covered with ribosomes than expected (Fig. 7F; Regions \#2 \& 3). Only region \#1 508 yielded detectable amplification products within the expected size range. Nonetheless, 
509 gel excision within the anticipated size ranges followed by cloning was performed for

510 all regions in all genotypes (Fig. S10A-C). Sanger sequencing revealed that 30 out of

51136 fragments cloned from region \#1 displayed 5'OH ends consistently mapping at

512 nucleotides 447-448, strikingly defining the intense ribosome stalling site on shGAG

513 (Fig. 7F, S10D) from which siRNA production is initiated (Fig. 7D). By contrast, the

514 clones obtained from regions \#2 and \#3 were either devoid of EVD sequences or

515 empty. These results are consistent with the notion that the intense ribosome stalling

516 event correlates with breakage of the shGAG RNA, and that the ensuing $5^{\prime} \mathrm{OH}$

517 fragments serve as templates for RDR6 to initiate dsRNA production and downstream

518 siRNA processing. Given that XRNs require a 5-P for their 5'->3' exonucleolytic

519 activities (Stevens, 2001; Schon et al, 2018), this could explain the insensitivity of

520 shGAG-derived siRNA accumulation to any $x r n$ mutation and to $x r n 4$ in particular

521 (Fig.6). Being linked to 3' cleavage fragments inaccessible to the competing activity of

522 XRN4, ribosome stalling might thus optimize the recruitment of RDR6 on shGAG for

523 PTGS initiation. We note that nanoPARE, while being indiscriminative of RNA 5'-ends

524 (including $5^{\prime} \mathrm{OH}$ ) requires a $3^{\prime}$ polyA tail to generate cDNA. Thus, the fact that the

525 technique failed to detect the shGAG $5^{\prime} \mathrm{OH}$ cleavage fragments could indicate that

526 they are indeed mostly poly(A)-. Preliminary PAGE-based analyses as conducted for

527 the 3' ends of ribothrypsis products in mammalian cells (Ibrahim et al. 2018) suggested

528 that discrete shGAG-derived 3' cleavage fragments are found in the poly $(A)^{-}$fraction

529 isolated from 35S:EVD in the rdr6 background (Fig.S11A). Lack-of-poly(A) could

530 further optimize RDR6 recruitment because the enzyme is inhibited in vitro by

531 3' adenosine stretches (Baeg et al, 2017). 


\section{Translation as an initiator of PTGS and epigenetic silencing}

536 Protein synthesis is commonly merely seen as a target of PTGS by reducing the

537 amount of available RNA and/or interfering with translation. Our study identifies

538 translation also as a trigger of PTGS. This became evident after epigenetic reactivation

539 of EVD, from which splicing-coupled PCPA generates separate RNA isoforms from a

540 single transcription unit. Of the two, the shorter subgenomic shGAG RNA undergoes

541 disproportionate translation over fIGAG-POL as an indispensable feature of Ty1/Copia

542 biology because this likely provides the stochiometric protein balance necessary for

543 efficient amplification and mobilization of the element. This process, however,

544 concomitantly stimulates RDR6 activity. shGAG translation efficacy per se is within

545 the range of moderately translated Arabidopsis mRNAs and is unlikely to explain this

546 effect, nor do GAG expression or abundance. Rather, an exceptionally intense and

547 highly discrete ribosome stalling event predisposes shGAG to RDR6-dependent

548 PTGS. Our data also suggest how intron-retention in combination with active splicing

549 accounts for the mostly nuclear versus cytosolic localization of fIGAG-POL versus

550 shGAG, respectively. Their asymmetrical subcellular distribution concurrently

551 rationalizes (i) the disproportionate translation efficacies of each mRNA, (ii) the

552 shGAG-centric distribution of translation-dependent EVD-derived siRNAs and,

553 consequently, (iii) the contrasted sensitivity of each isoform to cytosolic PTGS.

554 Splicing-coupled PCPA probably underlies most, if not all, of features $i$-iii because they

555 were recapitulated with the GFP-EVD $D_{\text {int/ter }}$ GUS construct containing the shGAG intron

556 and proximal PCPA signal (Fig.2, S4 and 5). Since splicing-coupled PCPA is at the

557 very core of the Ty1/Copia genome expression strategy (Oberlin et al, 2017), the

558 process described here for EVD is likely to be broadly applicable. 
Being mostly nuclear, fIGAG-POL, the template for RT required for mobilization,

560 is neither a potent trigger nor a target of PTGS, likely explaining why increasing 561 amounts of shGAG siRNAs have little impact on EVD's genomic proliferation over 562 successive epi15 inbred generations (Marí Ordóñez et al, 2013). Previously attributed 563 to GAG-mediated protection of fIGAG-POL as part of VLPs (Marí Ordóñez et al, 2013), 564 we now consider fIGAG-POL nuclear retention as an additional and perhaps major 565 contributor to this shielding effect. The ensuing rise in EVD genomic copies causes increasing levels of RDR6-dependent shGAG dsRNA over generations. We previously suggested that these levels eventually saturate DCL4/DCL2 activities in the highly cellspecific expression domain of EVD, acting as a prerequisite to DCL3 recruitment and

569 RdDM, ultimately causing LTR methylation and TGS of all EVD copies (Marí Ordóñez et al, 2013). This proposed saturation-coupled PTGS-to-TGS switch invariably occurs

571 in epi15 and other EVD-reactivating epiRILs when the EVD copy number reaches 4057250 (Marí Ordóñez et al, 2013), causing only sporadic and minor developmental defects 573 even in advanced generations (Marí Ordóñez et al, 2013; Mirouze et al, 2009; 574 Quadrana et al, 2016). By contrast, EVD copy number increases well beyond 80 in 575 rdr6 mutants already in F2s (Fig.6H) displaying loss-of-fertility (Fig.S11B) likely solely 576 ascribable to enhanced EVD proliferation. These data attests to a central role for 577 RDR6 in controlling EVD's mobilization and perhaps that of other autonomous TEs, at 578 the level of translation. At least in the multi-generational context of epi15, our results 579 also establish a hitherto unrecognized role for translation as an initiator of not only 580 PTGS, but also, ultimately, of epigenetic silencing and TGS. 
584 The vast majority of $d d m 1$-reactivated TEs that spawn RDR6-dependent siRNAs is composed of LTR/Gypsy elements (Fig.4), which is the family most prominently associated with easiRNA production (Creasey et al, 2014; Borges et al, 2018).

587 Arabidopsis LTR/Gypsy elements generally display significantly shorter-than-full588 length ORFs as compared to the other main classes of Arabidopsis TEs, including the $589 L T R /$ Copia family to which EVD belongs (Oberlin et al, 2017) (Fig.4C). Although they 590 likely constitute, therefore, degenerated transcription units, a substantial fraction of 591 such LTR/Gypsy is nonetheless highly expressed as a possible source of abundant 592 aberrant RNAs (Fig.4B). Thus, alternatively or concurrently to easiRNA production, 593 some of these LTR/Gypsy remnants might also enter the RDR6 pathway by saturating 594 RQC either co-transcriptionally or post-transcriptionally. While this process possibly 595 underlies a previously documented expression-dependent form of innate TE silencing 596 (Panda et al, 2016; Fultz \& Slotkin, 2017), such loci might in turn autonomously 597 produce siRNAs and become sources of identity-based silencing. Regardless, the 598 combined action of all these silencing pathways likely explains why most siRNA599 generating TEs in $d d m 1$ do not actively engage translation, as evidenced by their 600 conspicuous underrepresentation on polysomes (Fig.4A; quartile 4) (Oberlin et al, 601 2017). In fact, siRNA-generating TEs are equally or even less polysome-associated 602 than are non-coding RNAs (Fig.4A), indicating that there is no general correlation 603 between siRNA production and translation. Conversely, numerous TEs are translated, 604 yet do not spawn siRNAs (Fig.4A). These data contradict recent claims advocating a 605 general correlation between siRNA production and translation based on the untested 606 premise that most siRNA-generating TEs are translated (Kim et al, 2021), whereas 607 they are, in fact, absent from polysomes (Oberlin et al, 2017) (Fig.4A). Based on our 608 experimental findings we argue, on the contrary, that the process of "translation- 
609 dependent silencing" (TdS) described here is an attribute of only a handful of

610 evolutionary young TEs. These chiefly include EVD, which concurrently undergoes

611 productive translation (mostly of shGAG) and spawns RDR6-dependent siRNAs

612 (Figs.1, 4 and S1).

$613 \quad E V D$ is among the few autonomously transposing LTR/TEs in the Arabidopsis

614 Col-0 genome (Mirouze et al, 2009; Tsukahara et al, 2010; Gilly et al, 2014; Reinders

615 et al, 2009a) and, as such, is unlikely controlled by identity-based mechanisms. TdS

616 might enable the plant to detect its activity as the first line of defense against de novo

617 invasions, for instance upon horizontal transfer of active TEs. TdS may likewise

618 underpin silencing triggered upon experimental transfer of "exogenous" TEs between

619 species separated by millions of years of evolution (Hirochika et al, 2000; Fultz \&

620 Slotkin, 2017). Viruses divert a substantial fraction of the host translational apparatus

621 to their highly compact and TE-like genomic and subgenomic RNAs (GAO, 2003;

622 Dreher \& Miller, 2006; Sztuba-Solińska et al, 2011), which might also predispose them

623 to TdS. In all these circumstances, a key feature of TdS is an innate ability to detect

624 transcripts by virtue of their foreign - as opposed to aberrant - nature, independently

625 of any sequence homology to the host genome. We propose that foreignness is

626 perceived by anomalies manifested during active translation, which likely include

627 abnormal ribosome stalling, as discussed below.

628

629 Possible cause(s) of ribosome stalling

630 Our findings raise the key question of what molecular signal(s) might cause the 631 unusually intense stalling events observed in the shGAG and shGFP ORFs that 632 possibly activate TdS. While studies of ribosome stalling in plants are very scarce, 633 suboptimal codon usage has been widely considered as one of its possible causes 
634 (Rocha, 2004; Quax et al, 2015). Indeed, extensive stretches of rare codons trigger

635 ribosome stalling and RNA degradation when artificially engineered in reporter

636 mRNAs (Li et al, 2006; Yang et al, 2019; Park \& Subramaniam, 2019). However, it has

637 been noted that this rarely applies to endogenous mRNAs, where non-optimal codons

638 are common and play roles in translation regulation (Hanson \& Coller, 2018; Carneiro

639 et al, 2019). Accordingly, recent in-depth analyses in animals and fungi indicate that

640 codon usage per se is not predictive of stalling on endogenous mRNAs, and that other

641 factors might modulate ribosome dwell times (Gardin et al, 2014; Dana \& Tuller, 2014;

642 Rodnina, 2016; Zhang et al, 2017; Wu et al, 2019). shGAG and shGFP are devoid of

643 rare codons among other inspected features, suggesting that no universal

644 sequence/structure feature might underpin the stalling events correlating with TdS.

645 Rather, mRNA-intrinsic and variable features, or combinations thereof, might be

646 involved. mRNA-extrinsic features could also contribute to intense and discrete stalling

647 in shGAG via trans-interactions involving specific RNA sequence-motifs and RNA-

648 binding proteins (RBP), a circumstance that can hinder ribosome progression

649 (Babitzke et al, 2009; Iwakawa \& Tomari, 2015; Zhang et al, 2017). The action of AGO-

650 miRNA complexes could illustrate how RBPs may, under some circumstances, elicit

651 TdS, for instance during the intricate biogenesis of TAS3 trans-acting (ta)siRNAs (Hou

652 et al, 2016; Xia et al, 2017). We note that a recent model proposed by Kim and

653 coworkers (Kim et al, 2021) contends that PTGS via RDR6 might be caused by a

654 multitude of presumptive translation stalling events. These were allegedly ascribed to 655 pervasive suboptimal codons and low content in CG and CG3 along the ORFs of 656 certain mRNAs, including TE-derived RNAs. However, this interpretation is not 657 compatible with the highly discrete nature of the stalling events experimentally 
detected in our study and the noticeable absence from polysomes of the TE RNAs used to build this model, EVD excepted (Fig.4A).

660

\section{Possible mechanism(s) of TdS}

662 Intense stalling is usually resolved on the protein side by ubiquitin-mediated

663 proteolysis (Joazeiro, 2019). On the RNA side, it is released by translation-decoupled 664 RNA degradation related to, albeit distinct from, co-translational decay (Ikeuchi et al, 665 2018). One such process is XRN-mediated exonucleolysis operating in dedicated 666 processing $(\mathrm{P})$ bodies (Maldonado-Bonilla, 2014), yet 5'OH mRNA fragments are not 667 directly accessible to XRN action, which requires 5-P termini (Stevens, 2001). In 668 budding yeast, the Trl1 kinase progressively phosphorylates these fragments to 669 license their degradation by XRNs (Navickas et al, 2020), a process also likely 670 occurring during ribothrypsis in mammalian cells (D'Orazio et al, 2019). Alternatively,

671 or additively, the mouse and fission yeast DXO/Rai1, which removes incomplete 5'672 mRNA caps, catalyzes the removal of 5'OH ends, exposing 5' $\mathrm{P}$ for subsequent $5^{\prime}->3^{\prime}$ 673 exoribonuclease activity (Doamekpor et al, 2020). An Arabidopsis DXO1 catalytic 674 mutant for cap surveillance also displays such an activity in vitro, although it shows 675 higher affinity for 5'P substrates (Doamekpor et al, 2020). Whether AtDXO1 antagonizes TdS in vivo will have to be further investigated. In contrast to RNAi-deficient budding yeast or RNAi-proficient mammalian cells, 678 plants display RDR activities (Stein et al, 2003; Drinnenberg et al, 2009). We suggest 679 that in these organisms, $5^{\prime} \mathrm{OH}$ termini would not only disqualify XRN4 action, but 680 concurrently optimize that of RDR6, which is known to compete with XRN4 for 
et al, 2008). Incidentally, 5'P -termini are also generated during mRNA decapping (Hu

et al, 2009), potentially explaining, for similar reasons, why decapping Arabidopsis

TdS is possibly further facilitated by the striking physical proximity of P-bodies - where

unresolved $5^{\prime} \mathrm{OH}$ RNA fragments should primarily accumulate - with the so-called

"siRNA bodies" (Martínez de Alba et al, 2015). RDR6 and co-factors congregate in

these bodies for tasiRNA processing, which, we suggest, are also the sites of shGAG

siRNA biogenesis. We emphasize that converting the $5^{\prime} \mathrm{OH}-\mathrm{RNA}$ fragments into

dsRNA would lead to siRNA production accompanied by limited exonucleolytic

degradability of RNA. Widespread RNA degradation intermediates spanning entire

692 transcriptional units were recently proposed to represent RDR6 substrates for

693 translation-coupled PTGS including in the case of EVD (Kim et al, 2021). This model,

694 however, does not explain the discrete siRNA pattern observed exclusively on shGAG

695 yet not fIGAG-POL. It neither explains why highly abundant, ORF-spanning RNA

696 degradation products, such as those derived from ACT2, do not correlate with siRNA 697 production.

Capped but poly(A)- 5' RNA fragments are expected to be concurrently

site on shGAG. Unlike the XRN4-protected 5'OH 3' fragments, however, these

7025 ' fragments would be a priori devoid of features preventing their SKI2-mediated 703 exosomal 3'-5' degradation, which indeed can outcompete RDR6 action (Zhang et al,

704 2015; Branscheid et al, 2015). Differential competition between SKI2-mediated 705 degradation versus RDR6 activity, as previously reported for some miRNA targets 706 (Branscheid et al, 2015), might explain why the siRNAs pattern from shGFP spans 
both the 5' and 3' sequences surrounding the identified stalling site whereas these are

708 only located 3' to the shGAG stalling site of (Fig.7D, S8E). In principle, RDR6 could also pick up a multitude of RNA cleavage fragments predictably produced via siRNA-

710 guided cleavage of shGAG by AGO1/AGO2-RISCs. However, RISC-mediated slicing

711 produces 5'P termini (Martinez \& Tuschl, 2004), which would qualify these RNAs as

712 XRN4-, as opposed to RDR6-, substrates, therefore unlikely contributing prominently

713 to shGAG siRNA production. A final, outstanding aspect of TdS pertains to the

714 mechanism whereby $5{ }^{\prime} \mathrm{OH}$ fragments are generated. In budding yeast, the metal-

715 independent endonuclease Cue2 was recently shown to cleave, within the colliding

716 ribosome's A site, mRNAs undergoing stalling-induced no-go decay, which generates

717 5'OH 3' RNA fragments (D'Orazio et al, 2019). The mammalian homolog, N4BP2,

718 which additionally contains a polynucleotide kinase domain, might directly couple

719 endonucleolysis with the 5'P-dependent XRN-licensing step evoked above (D'Orazio

720 et al, 2019). We failed, however, to identify a plant Cue2/ N4BP2 ortholog. We suggest

721 that other mechanisms might underlie what we therefore conservatively refer to as

722 "translation-linked mRNA breakage" here. EVD or reporters derived thereof (e.g. GFP-

$723 E V D_{\text {int/ter }}$ GUS) might provide a paradigm to elucidate, via biochemistry and forward

724 genetics, the mechanisms of TdS in plants.

Among other processes, transient ribosome stalling is a normal and favorable

726 feature of translation enabling proper folding of nascent peptides (Rodnina, 2016).

727 Accordingly, many mechanisms exist to resolve such instances (Buskirk \& Green, 728 2017) including ribothrypsis in mammalian cells, which appears to be a widespread 729 component of ordinary translation (Ibrahim et al, 2018). Like ribothrypsis, the process 730 described here might have eluded characterization for years because its products 731 have 5'OH and possible 3' poly(A)- termini inaccessible to standard RNA sequencing 
732 procedures. However, while its initiation strongly resembles that of mammalian

733 ribothrypsis, TdS is unlikely to be ubiquitous in plants, since the aforementioned RNA

734 products, by directly engaging RDR6 for amplified siRNA production, would promote

735 degradation of the entire mRNA pool independently of its stalled or even merely

736 translated status. While this would be highly detrimental as a common form of

737 endogenous gene regulation, the process seems particularly well-suited to eliminate

738 highly proliferating foreign RNAs such as those of viruses and TEs. We suspect that

739 TdS might also represent a yet unexplored trigger for PTGS of transgenes encoding,

740 in particular, non-plant ORFs with suboptimal translation features.

\section{Author contributions:}

742 A.M.O., S.O., and O.V. conceived and designed the study. S.O. and A.M.O. performed

743 most experiments. R.R. cloned and sequenced the EVD 5'OH ends, M.T. and V.B.B.

744 investigated EVD copy number in rdr6 and RNA isoform distribution in cyto-nuclear

745 fractions. M.A.S., A.P. and M.N. performed nanoPARE. L.L. conducted seed counting.

746 S.O. and M.A.S performed computer analyses. S.O. and M.T performed statistical

747 analyses. A.M.O., S.O., and O.V. analyzed the data and wrote the manuscript.

\section{Acknowledgements:}

749 We thank members of the Voinnet and Marí-Ordóñez labs and colleagues for critical

750 reading of the manuscript and for discussions. This work was supported by a core

751 grant attributed to OV by the ETH-Zürich that covered the largest part of this study,

752 including the $\mathrm{PhD}$ studentships of S.O. and A.M.O. under the Life Science Zürich

753 Graduate School program. Part of the work was also supported by the NCCR RNA \&

754 Disease funded by the Swiss National Science Foundation. 
bioRxiv preprint doi: https://doi.org/10.1101/2021.06.29.450179; this version posted June 29, 2021. The copyright holder for this preprint (which was not certified by peer review) is the author/funder, who has granted bioRxiv a license to display the preprint in perpetuity. It is made available under aCC-BY 4.0 International license.

\section{Competing interests:}

756 The authors declare no competing interests. 


\section{REFERENCES}

758 Allshire RC \& Madhani HD (2018) Ten principles of heterochromatin formation and function. Nature Reviews Molecular Cell Biology 19: 229-244

Artieri CG \& Fraser HB (2014) Accounting for biases in riboprofiling data indicates a major role for proline in stalling translation. Genome Research 24: 2011-2021

Babitzke P, Baker CS \& Romeo T (2009) Regulation of translation initiation by RNA binding proteins. Annu Rev Microbiol 63: 27-44

Baeg K, Iwakawa H-O \& Tomari Y (2017) The poly(A) tail blocks RDR6 from converting self mRNAs into substrates for gene silencing. Nat Plants 3: 17036-4

Bao C, Loerch S, Ling C, Korostelev AA, Grigorieff N \& Ermolenko DN (2020) mRNA stem-loops can pause the ribosome by hindering A-site tRNA binding. eLife 9:

Borges F, Parent J-S, Van Ex F, Wolff P, Martínez G, Köhler C \& Martienssen RA (2018) Transposonderived small RNAs triggered by miR845 mediate genome dosage response in Arabidopsis. Nat Genet 50: 186-192

771 Branscheid A, Marchais A, Schott G, Lange H, Gagliardi D, Andersen SU, Voinnet O \& Brodersen P (2015) SKI2 mediates degradation of RISC 5'-cleavage fragments and prevents secondary siRNA production from miRNA targets in Arabidopsis. Nucleic Acids Research: gkv1014

774 Brodersen P \& Voinnet O (2006) The diversity of RNA silencing pathways in plants. Trends in Genetics 22: $268-280$

Buskirk AR \& Green R (2017) Ribosome pausing, arrest and rescue in bacteria and eukaryotes. Philos.

778 Carneiro RL, Requião RD, Rossetto S, Domitrovic T \& Palhano FL (2019) Codon stabilization coefficient as a metric to gain insights into mRNA stability and codon bias and their relationships with translation. Nucleic Acids Research 47: 2216-2228

Civáň P, Švec M \& Hauptvogel P (2011) On the Coevolution of Transposable Elements and Plant 
D'Orazio KN, Wu CC-C, Sinha N, Loll-Krippleber R, Brown GW \& Green R (2019) The endonuclease Cue2 cleaves mRNAs at stalled ribosomes during No Go Decay. eLife 8:

Dalakouras A, Lauter A, Bassler A, Krczal G \& Wassenegger M (2019) Transient expression of introncontaining transgenes generates non-spliced aberrant pre-mRNAs that are processed into siRNAs. Planta 249: 457-468

Dana A \& Tuller T (2014) The effect of tRNA levels on decoding times of mRNA codons. Nucleic Acids Research 42: 9171-9181

793

Desai KK \& Raines RT (2012) tRNA ligase catalyzes the GTP-dependent ligation of RNA with 3phosphate and 5'-hydroxyl termini. Biochemistry 51: 1333-1335

795

Doamekpor SK, Gozdek A, Kwasnik A, Kufel J \& Tong L (2020) A novel 5'-hydroxyl dinucleotide hydrolase activity for the DXO/Rai1 family of enzymes. Nucleic Acids Research 48: 349-358

Dobin A, Davis CA, Schlesinger F, Drenkow J, Zaleski C, Jha S, Batut P, Chaisson M \& Gingeras TR (2013) STAR: ultrafast universal RNA-seq aligner. Bioinformatics 29: 15-21

Doma MK \& Parker R (2006) Endonucleolytic cleavage of eukaryotic mRNAs with stalls in translation elongation. Nature 440: $561-564$

801 Doma MK \& Parker R (2007) RNA quality control in eukaryotes. Cell 131: 660-668

802 Dreher TW \& Miller WA (2006) Translational control in positive strand RNA plant viruses. Virology 344: 185-197

804 Drinnenberg IA, Weinberg DE, Xie KT, Mower JP, Wolfe KH, Fink GR \& Bartel DP (2009) RNAi in budding yeast. Science 326: $544-550$

806 Dumesic PA, Natarajan P, Chen C, Drinnenberg IA, Schiller BJ, Thompson J, Moresco JJ, Yates JR III, Bartel DP \& Madhani HD (2013) Stalled Spliceosomes Are a Signal for RNAi-Mediated Genome Defense. Cell 152: 957-968

809 Fay MM, Lyons SM \& Ivanov P (2017) RNA G-Quadruplexes in Biology: Principles and Molecular Mechanisms. J. Mol. Biol. 429: 2127-2147

811 Fedoroff NV (2012) Transposable Elements, Epigenetics, and Genome Evolution. Science 338: 758812 767

813 Fultz D \& Slotkin RK (2017) Exogenous Transposable Elements Circumvent Identity-Based Silencing, 814 Permitting the Dissection of Expression-Dependent Silencing. THE PLANT CELL ONLINE 29: 360-376 
816 Gao X (2003) Translational recoding signals between gag and pol in diverse LTR retrotransposons.

RNA 9: $1422-1430$

818 Gardin J, Yeasmin R, Yurovsky A, Cai Y, Skiena S \& Futcher B (2014) Measurement of average 819 decoding rates of the 61 sense codons in vivo. elife 3:

820 Gazzani S (2004) A Link Between mRNA Turnover and RNA Interference in Arabidopsis. Science 306:

821 1046-1048

822 Gilly A, Etcheverry M, Madoui M-A, Guy J, Quadrana L, Alberti A, Martin A, Heitkam T, Engelen S, Labadie K, Le Pen J, Wincker P, Colot V \& Aury J-M (2014) TE-Tracker: systematic identification of transposition events through whole-genome resequencing. BMC Bioinformatics 15: 377

Gregory BD, O'Malley RC, Lister R, Urich MA, Tonti-Filippini J, Chen H, Millar AH \& Ecker JR (2008) A

826 Link between RNA Metabolism and Silencing Affecting Arabidopsis Development. Developmental

828 Gy I, Gasciolli V, Lauressergues D, Morel J-B, Gombert J, Proux F, Proux C, Vaucheret H \& Mallory AC (2007a) Arabidopsis FIERY1, XRN2, and XRN3 Are Endogenous RNA Silencing Suppressors. Plant Cell 19: 3451-3461

Hahne F \& Ivanek R (2016) Visualizing Genomic Data Using Gviz and Bioconductor. Methods Mol. Biol. 1418: $335-351$

833 Hanson G \& Coller J (2018) Codon optimality, bias and usage in translation and mRNA decay. Nature Reviews Molecular Cell Biology 19: 20-30

835 Herr AJ, Molnar A, Jones A \& Baulcombe DC (2006) Defective RNA processing enhances RNA silencing and influences flowering of Arabidopsis. Proceedings of the National Academy of

838 Hirochika H, Okamoto H \& Kakutani T (2000) Silencing of retrotransposons in arabidopsis and reactivation by the ddm1 mutation. Plant Cell 12: 357-368

840 Hou CY, Lee WC, Chou HC, Chen AP, Chou SJ \& Chen H-M (2016) Global Analysis of Truncated RNA

841 Ends Reveals New Insights into Ribosome Stalling in Plants. Plant Cell 28: 2398-2416

$842 \mathrm{Hu}$ W, Sweet TJ, Chamnongpol S, Baker KE \& Coller J (2009) Co-translational mRNA decay in 843 Saccharomyces cerevisiae. Nature 461: 225-229

844 Huang CRL, Burns KH \& Boeke JD (2012) Active Transposition in Genomes. Annu Rev Genet 46: 651- 
846 Ibrahim F, Maragkakis M, Alexiou P \& Mourelatos Z (2018) Ribothrypsis, a novel process of canonical 847 mRNA decay, mediates ribosome-phased mRNA endonucleolysis. Nature structural \& molecular 848 biology 25: 302-310

849 Ikeuchi K, Izawa T \& Inada T (2018) Recent Progress on the Molecular Mechanism of Quality Controls $850 \quad$ Induced by Ribosome Stalling. Front Genet 9: 743

851 Ingolia NT, Ghaemmaghami S, Newman JRS \& Weissman JS (2009) Genome-wide analysis in vivo of 852 translation with nucleotide resolution using ribosome profiling. Science 324: 218-223

853 Iwakawa H-O \& Tomari Y (2015) The Functions of MicroRNAs: mRNA Decay and Translational 854 Repression. Trends in Cell Biology 25: 651-665

855 Jia J, Long Y, Zhang H, Li Z, Liu Z, Zhao Y, Lu D, Jin X, Deng X, Xia R, Cao X \& Zhai J (2020) Post856 transcriptional splicing of nascent RNA contributes to widespread intron retention in plants. Nat

858 Joazeiro CAP (2019) Mechanisms and functions of ribosome-associated protein quality control. Nature Reviews Molecular Cell Biology 20: 368-383

860 Kankel MW, Ramsey DE, Stokes TL, Flowers SK, Haag JR, Jeddeloh JA, Riddle NC, Verbsky ML \& Richards EJ (2003) Arabidopsis MET1 cytosine methyltransferase mutants. Genetics 163: 1109_

862 1122

863

Kastenmayer JP \& Green PJ (2000) Novel features of the XRN-family in Arabidopsis: Evidence that AtXRN4, one of several orthologs of nuclear Xrn2p/Rat1p, functions in the cytoplasm. Proc. Natl. Acad. Sci. U.S.A. 97: 13985-13990

Khanduja JS, Calvo IA, Joh RI, Hill IT \& Motamedi M (2016) Nuclear Noncoding RNAs and Genome

868 Kim EY, Wang L, Lei Z, Li H, Fan W \& Cho J (2021) Ribosome stalling and SGS3 phase separation prime the epigenetic silencing of transposons. Nat Plants 7: 303-309

870 Kurihara Y (2017) Activity and roles of Arabidopsis thaliana XRN family exoribonucleases in noncoding RNA pathways. J Plant Res 130: 25-31

872 Langmead B \& Salzberg SL (2012) Fast gapped-read alignment with Bowtie 2. Nat Meth 9: 357-359

873 Lauria F, Tebaldi T, Bernabò P, Groen EJN, Gillingwater TH \& Viero G (2018) riboWaltz: Optimization 874 of ribosome P-site positioning in ribosome profiling data. PLoS Comp Biol 14: e1006169 
875 Lee ES, Akef A, Mahadevan K \& Palazzo AF (2015) The consensus 5' splice site motif inhibits mRNA $876 \quad$ nuclear export. PLOS ONE 10: e0122743

877 Lee SC, Ernst E, Berube B, Borges F, Parent J-S, Ledon P, Schorn A \& Martienssen RA (2020) 878 Arabidopsis retrotransposon virus-like particles and their regulation by epigenetically activated 879 small RNA. Genome Research 30: 576-588

880 Li H, Handsaker B, Wysoker A, Fennell T, Ruan J, Homer N, Marth G, Abecasis G, Durbin R1000 Genome Project Data Processing Subgroup (2009) The Sequence Alignment/Map format and SAMtools. Bioinformatics 25: 2078-2079

Li X, Hirano R, Tagami H \& Aiba H (2006) Protein tagging at rare codons is caused by tmRNA action at the $3^{\prime}$ end of nonstop mRNA generated in response to ribosome stalling. RNA 12: 248-255

Liao Y, Smyth GK \& Shi W (2013) The Subread aligner: fast, accurate and scalable read mapping by seed-and-vote. Nucleic Acids Research 41: e108

Llave C, Xie Z, Kasschau KD \& Carrington JC (2002) Cleavage of Scarecrow-like mRNA targets directed by a class of Arabidopsis miRNA. Science 297: 2053-2056

890

Love MI, Huber W \& Anders S (2014) Moderated estimation of fold change and dispersion for RNAseq data with DESeq2. Genome Biol 15: 550-21

Luo Z \& Chen Z (2007) Improperly terminated, unpolyadenylated mRNA of sense transgenes is targeted by RDR6-mediated RNA silencing in Arabidopsis. Plant Cell 19: 943-958

Maldonado-Bonilla LD (2014) Composition and function of P bodies in Arabidopsis thaliana. Front. Plant Sci. 5: 201

Marí Ordóñez A, Marchais A, Etcheverry M, Martin A, Colot V \& Voinnet O (2013) Reconstructing de novo silencing of an active plant retrotransposon. Nat Genet 45: 1029-1039

Martinez J \& Tuschl T (2004) RISC is a 5' phosphomonoester-producing RNA endonuclease. Genes

899 Martínez de Alba AE, Moreno AB, Gabriel M, Mallory AC, Christ A, Bounon R, Balzergue S, Aubourg 900 S, Gautheret D, Crespi MD, Vaucheret H \& Maizel A (2015) In plants, decapping prevents RDR6- 
Moreno AB, Martínez de Alba AE, Bardou F, Crespi MD, Vaucheret H, Maizel A \& Mallory AC (2013) Cytoplasmic and nuclear quality control and turnover of single-stranded RNA modulate posttranscriptional gene silencing in plants. Nucleic Acids Research 41: 4699-4708 Available at: http://www.nar.oxfordjournals.org/cgi/doi/10.1093/nar/gkt152

Navickas A, Chamois S, Saint-Fort R, Henri J, Torchet C \& Benard L (2020) No-Go Decay mRNA

Nuthikattu S, McCue AD, Panda K, Fultz D, DeFraia C, Thomas EN \& Slotkin RK (2013) The Initiation of Epigenetic Silencing of Active Transposable Elements Is Triggered by RDR6 and 21-22 Nucleotide Small Interfering RNAs. Plant Physiology 162: 116-131

Oberlin S, Sarazin A, Chevalier C, Voinnet O \& Marí Ordóñez A (2017) A genome-wide transcriptome and translatome analysis of Arabidopsis transposons identifies a unique and conserved genome expression strategy for Ty1/Copia retroelements. Genome Research 27: 1549-1562

920

Panda K, Ji L, Neumann DA, Daron J, Schmitz RJ \& Slotkin RK (2016) Full-length autonomous transposable elements are preferentially targeted by expression-dependent forms of RNA-directed DNA methylation. Genome Biol 17: 170-19

Parent J-S, Jauvion V, Bouché N, Beclin C, Hachet M, Zytnicki M \& Vaucheret H (2015) Posttranscriptional gene silencing triggered by sense transgenes involves uncapped antisense RNA and differs from silencing intentionally triggered by antisense transgenes. Nucleic Acids Research 43: $8464-8475$

Park H \& Subramaniam AR (2019) Inverted translational control of eukaryotic gene expression by

928 Peach SE, York K \& Hesselberth JR (2015) Global analysis of RNA cleavage by 5'-hydroxyl RNA 929 sequencing. Nucleic Acids Research 43: gkv536-e108

930 Pelechano V, Wei W \& Steinmetz LM (2015) Widespread Co-translational RNA Decay Reveals $931 \quad$ Ribosome Dynamics. Cell 161: 1400-1412 
932 Peragine A, Yoshikawa M, Wu G, Albrecht HL \& Poethig RS (2004) SGS3 and SGS2/SDE1/RDR6 are required for juvenile development and the production of trans-acting siRNAs in Arabidopsis. Genes Dev. 18: 2368-2379

935 Popa A, Lebrigand K, Paquet A, Nottet N, Robbe-Sermesant K, Waldmann R \& Barbry P (2016) 936 RiboProfiling: a Bioconductor package for standard Ribo-seq pipeline processing. F1000Research 5: 1309

938 Quadrana L, Bortolini Silveira A, Mayhew GF, LeBlanc C, Martienssen RA, Jeddeloh JA \& Colot V 939 (2016) The Arabidopsis thaliana mobilome and its impact at the species level. eLife 5:

940 Quax TEF, Claassens NJ, Söll D \& van der Oost J (2015) Codon Bias as a Means to Fine-Tune Gene Expression. Molecular Cell 59: 149-161

942 Quinlan AR \& Hall IM (2010) BEDTools: a flexible suite of utilities for comparing genomic features.

944 Reinders J, Wulff BBH, Mirouze M, Marí Ordóñez A, Dapp M, Rozhon W, Bucher E, Theiler G \& 945 Paszkowski J (2009a) Compromised stability of DNA methylation and transposon immobilization in mosaic Arabidopsis epigenomes. Genes Dev. 23: 939-950

947 Rocha EPC (2004) Codon usage bias from tRNA's point of view: redundancy, specialization, and 948 efficient decoding for translation optimization. Genome Research 14: 2279-2286

949 Rodnina MV (2016) The ribosome in action: Tuning of translational efficiency and protein folding. $950 \quad$ Protein Sci 25: 1390-1406

951 Sabi R \& Tuller T (2015) A comparative genomics study on the effect of individual amino acids on 952 ribosome stalling. BMC Genomics 16 Suppl 10: S5-12

953 Sarazin A \& Voinnet O (2014) Exploring new models of easiRNA biogenesis. Nat Genet 46: 530-531

954 Saze H, Scheid OM \& Paszkowski J (2003) Maintenance of CpG methylation is essential for epigenetic inheritance during plant gametogenesis. Nat Genet 34: 65-69

956 Schon MA, Kellner MJ, Plotnikova A, Hofmann F \& Nodine MD (2018) NanoPARE: parallel analysis of RNA 5' ends from low-input RNA. Genome Research 28: 1931-1942

958 Silber R, Malathi VG \& Hurwitz J (1972) Purification and properties of bacteriophage T4-induced RNA ligase. Proc. Natl. Acad. Sci. U.S.A. 69: 3009-3013

960 Song J, Perreault J-P, Topisirovic I \& Richard S (2016) RNA G-quadruplexes and their potential regulatory roles in translation. Translation (Austin) 4: e1244031 
962 Stein P, Svoboda P, Anger M \& Schultz RM (2003) RNAi: mammalian oocytes do it without RNAdependent RNA polymerase. RNA 9: 187-192

964 Stevens A (2001) 5'-exoribonuclease 1: Xrn1. Methods in enzymology 342: 251-259

965 Sztuba-Solińska J, Stollar V \& Bujarski JJ (2011) Subgenomic messenger RNAs: mastering regulation 966 of (+)-strand RNA virus life cycle. Virology 412: 245-255

967 Sørensen BB, Ehrnsberger HF, Esposito S, Pfab A, Bruckmann A, Hauptmann J, Meister G, MerkI R, 968 Schubert T, Längst G, Melzer M, Grasser M \& Grasser KD (2017) The Arabidopsis THO/TREX component TEX1 functionally interacts with MOS11 and modulates mRNA export and alternative splicing events. Plant Mol Biol 93: 283-298

971 Šurbanovski N, Brilli M, Moser M \& Si-Ammour A (2016) A highly specific microRNA-mediated 972 mechanism silences LTR retrotransposons of strawberry. The Plant Journal 85: 70-82

973 Teixeira FK, Heredia F, Sarazin A, Roudier F, Boccara M, Ciaudo C, Cruaud C, Poulain J, Berdasco M, Fraga MF, Voinnet O, Wincker P, Esteller M \& Colot V (2009) A Role for RNAi in the Selective Correction of DNA Methylation Defects. Science 323: 1600-1604

Thran M, Link K \& Sonnewald U (2012) The Arabidopsis DCP2gene is required for proper mRNA

978 Tsukahara S, Kobayashi A, Kawabe A, Mathieu O, Miura A \& Kakutani T (2010) Bursts of retrotransposition reproduced in Arabidopsis. Nature 461: 423-426

980 Valencia P, Dias AP \& Reed R (2008) Splicing promotes rapid and efficient mRNA export in mammalian cells. Proceedings of the National Academy of Sciences 105: 3386-3391

982 Vazquez F, Vaucheret H, Rajagopalan R, Lepers C, Gasciolli V, Mallory AC, Hilbert JL, Bartel DP \& Crete P (2004) Endogenous trans-acting siRNAs regulate the accumulation of Arabidopsis mRNAs. Molecular cell 16: 69-79

Vitte C \& Bennetzen JL (2006) Analysis of retrotransposon structural diversity uncovers properties and propensities in angiosperm genome evolution. Proc. Natl. Acad. Sci. U.S.A. 103: 17638-17643

Vitte C \& Panaud O (2005) LTR retrotransposons and flowering plant genome size: emergence of the increase/decrease model. Cytogenet Genome Res 110: 91-107 
Vongs A, Kakutani T, Martienssen R \& Richards E (1993) Arabidopsis thaliana DNA methylation mutants. Science 260: 1926-1928

Wang C \& Fang J (2015) RLM-RACE, PPM-RACE, and qRT-PCR: an integrated strategy to accurately validate miRNA target genes. Methods Mol. Biol. 1296: 175-186

995 Wickham H ggplot2 - Elegant Graphics for Data Analysis. Springer.

996 Wu CC-C, Zinshteyn B, Wehner KA \& Green R (2019) High-Resolution Ribosome Profiling Defines Molecular Cell 73: 959-970.e5

1000

Xia R, Xu J \& Meyers BC (2017) The Emergence, Evolution, and Diversification of the miR390-TAS3ARF Pathway in Land Plants. Plant Cell 29: 1232-1247

1002

Yan S, Wen J-D, Bustamante C \& Tinoco I (2015) Ribosome excursions during mRNA translocation

1003 mediate broad branching of frameshift pathways. Cell 160: 870-881

1004

Yang Q, Yu C-H, Zhao F, Dang Y, Wu C, Xie P, Sachs MS \& Liu Y (2019) eRF1 mediates codon usage

1005 effects on mRNA translation efficiency through premature termination at rare codons. Nucleic Acids

1006 Research 47: 9243-9258

1007

Yin Y, Lu JY, Zhang X, Shao W, Xu Y, Li P, Hong Y, Cui L, Shan G, Tian B, Zhang QC \& Shen X (2020)

1008 U1 snRNP regulates chromatin retention of noncoding RNAs. Nature 580: 147-150

1009

1010

Yu X, Willmann MR, Anderson SJ \& Gregory BD (2016) Genome-Wide Mapping of Uncapped and

Zemach A, Kim MY, Hsieh P-H, Coleman-Derr D, Eshed-Williams L, Thao K, Harmer SL \& Zilberman Cleaved Transcripts Reveals a Role for the Nuclear mRNA Cap-Binding Complex in

Zhang S, Hu H, Zhou J, He X, Jiang T \& Zeng J (2017) Analysis of Ribosome Stalling and Translation Elongation Dynamics by Deep Learning. Cell Syst 5: 212-220.e6

1016

Zhang X, Zhu Y, Liu X, Hong X, Xu Y, Zhu P, Shen Y, Wu H, Ji Y, Wen X, Zhang C, Zhao Q, Wang Y, Lu J \& Guo H (2015) Plant biology. Suppression of endogenous gene silencing by bidirectional 1019 Zhao T, Chen Y-M, Li Y, Wang J, Chen S, Gao N \& Qian W (2021) Disome-seq reveals widespread ribosome collisions that promote cotranslational protein folding. Genome Biol 22: 16-35 


\section{Material and methods:}

\section{Plant material and growth conditions}

1023 Plants were grown in a growth chamber on soil at $22^{\circ} \mathrm{C}$ for two weeks in a $12 \mathrm{~h} / 12 \mathrm{~h}$ 1024 light cycle and then transferred to a 16h/8h light cycle and pools of three to five plants 1025 were sampled for inflorescence tissue. Mutant genotypes met1-3, dc/1-11, ddm1-2 1026 (seventh inbred generation), hyl1-2, rdr6-12, xrn2-2, xrn3-3, xrn4-3 plants are all 1027 derived from the Col-0 ecotype (Vongs et al, 1993; Peragine et al, 2004; Vazquez et 1028 al, 2004; Gy et al, 2007b). Genotyping primers are described in Suppl. Table 1. met11029 derived epiRIL\#15 plants (epi15) were described previously (Reinders et al, 2009b; 1030 Oberlin et al, 2017). 35S:EVDwt, 35S:EVD $m$ U1, 35S:EVD $D_{\Delta i}, 35 S: G F P-G U S$ and 1031 35S:GFP-EVD intter-GUS overexpression lines were previously depicted (Marí 1032 Ordóñez et al, 2013; Oberlin et al, 2017).

1033

1034 Constructs and Plasmids:

1035 All constructs are available from addgene (www.addgene.org): 35S:EVDwt (\#167119), 35S:EVDmU1 (\#167121), 35S:EVDAI (\#167120), 35S:GFP-GUS (\#167122) and 35S:GFP-EVDint/ter-GUS (\#167123) (Marí Ordóñez et al, 2013; Oberlin et al, 2017).

1038

1039 Cyto-nuclear fractionations:

1040 For each sample, twice $250 \mathrm{mg}$ of 3 week-old seedlings grown in $1 / 2$ strength ( $2.2 \mathrm{~g} / \mathrm{L})$

1041 Murashige and Skoog medium (\#M0231,Duchefa Biochemie) were ground to fine 1042 powder in liquid nitrogen and homogenized in $575 \mu \mathrm{L}$ of lysis buffer (10 mM Tris- $\mathrm{HCl}$ $1043 \mathrm{pH} 7.4,150 \mathrm{mM} \mathrm{NaCl}, 0.15 \%$ IGEPAL (CA-630, Merk) and 1x cOmplete protease 1044 inhibitor cocktail (Roche)). Lysates were gently mixed and incubated on ice for $10 \mathrm{~min}$. 
1045 before being filtered through one layer of Miracloth. $400 \mu \mathrm{L}$ from each lysate were 1046 recovered and one set aside as Total. The second set of cell lysate were gently 1047 overlaid on top of $1 \mathrm{~mL}$ of cold sucrose buffer $(10 \mathrm{mM}$ Tris- $\mathrm{HCl} \mathrm{pH} \mathrm{7.4,150} \mathrm{mM} \mathrm{NaCl}$, $104824 \%$ sucrose and 1x cOmplete EDTA-free protease inhibitor cocktail (\#04693159001, 1049 Roche)) in protein low binding $1.5 \mathrm{~mL}$ tubes (LoBind, Eppendorf) by slowly pipetting 1050 against the side of the tube. Samples were centrifuged at $3500 \mathrm{x}, \mathrm{g}$ for $10 \mathrm{~min}$. to separate nuclei (pellet) from cytoplasm (supernatant). Cytoplasmic fractions were cleared by centrifugation at $14000 \times \mathrm{g}$ for $1 \mathrm{~min}$. in a new tube and the resulting 1053 supernatant set aside. Nuclear pellets were rinsed by inverting the tube 3-5 times 1054 without disturbing the pellet with $1 \mathrm{~mL}$ of $1 \mathrm{X}$ PBS, $0.5 \mathrm{mM}$ EDTA. Nuclei were spin for 15 s. at $1300 \times g$ before gently removing the wash solution. Nuclei pellets were resuspended by pipetting in $200 \mu \mathrm{L}$ of nuclear lysis buffer $(10 \mathrm{mM}$ Tris- $\mathrm{HCl} \mathrm{pH} 7.4$, $300 \mathrm{mM} \mathrm{NaCl}, 7.5 \mathrm{mM} \mathrm{MgCl}_{2}, 0.2 \mathrm{mM}$ EDTA pH8, 1M Urea, 1\% IGEPAL and 1x cOmplete protease inhibitor cocktail). For isolation of total RNA and protein from the 1059 different fractions, samples were mixed 1 volume of acid $\mathrm{PCl}$ 1060 (Phenol/Chloroform/Isoamyl-alcohol, \#X985 Carl Roth). In addition, nuclear fractions 1061 were further homogenized after addition of PCI by passing the sample through a 21gauge needle with a $1 \mathrm{~mL}$ syringe. All steps were carried on ice or centrifuged at $4^{\circ} \mathrm{C}$.

1063 Buffers were freshly prepared in advance and chilled on ice before use.

\section{Nucleic acid and protein extractions}

RNA was extracted from frozen and ground tissue with TRIzol reagent (\#93289,

1067 Sigma) and precipitated with $1 \times$ vol. of cold isopropanol. For RNA extraction from cyto1068 nuclear fractionations, $20 \mu \mathrm{g}$ of glycogen (\#R0551, ThermoFisher) and 0.1x vol. of 1069 sodium acetate $3 \mathrm{M}$ pH5.2 were mixed with recovered aqueous phases after $\mathrm{PCl}$ 
1070

1071

1072

1073

1074

1075

1076

1077

1078

1079

1080

1081

1082

\section{3}

1084

1085

1086

1087

1088

1089

1090

1091

1092

1093

1094

before RNA precipitation with $1 \mathrm{x}$ vol. of cold isopropanol. DNA was extracted using the DNeasy Plant Mini Kit (\#69204, Qiagen) according to manufacturer's guidelines.

Protein of frozen and ground tissue was homogenized in extraction buffer $(0.7 \mathrm{M}$ sucrose, 0.5 M Tris- $\mathrm{HCl}$, pH 8, 5 mM EDTA, pH 8, $0.1 \mathrm{M} \mathrm{NaCl}, 2 \% \beta$-mercaptoethanol) and cOmplete EDTA-free protease inhibitor cocktail (\#04693159001, Roche). Watersaturated and Tris-buffered phenol $(\mathrm{pH} 8)$ was added to an equal volume and samples were agitated for $5 \mathrm{~min}$. Phases were separated by $30 \mathrm{~min}$ centrifugation $(12,000 \mathrm{~g}$ at $4{ }^{\circ} \mathrm{C}$ ). Proteins were precipitated from the phenol phase (including those from $\mathrm{PCl}$ ) by the addition of 5 volumes of $0.1 \mathrm{M}$ ammonium acetate in methanol. Precipitated proteins were collected by centrifugation for $30 \mathrm{~min}\left(12,000 \mathrm{~g}\right.$ at $\left.4{ }^{\circ} \mathrm{C}\right)$, washed twice with ammonium acetate in methanol and resuspended in resuspension buffer $(3 \%$ SDS, $62.3 \mathrm{mM}$ Tris- $\mathrm{HCl}, \mathrm{pH} 8,10 \%$ glycerol).

\section{RNA and protein blot analysis}

For high molecular weight RNA analysis, $5-10 \mu \mathrm{g}$ of total RNA was separated on a $1.2 \%$ agarose MOPS-buffered gel with $2.2 \mathrm{M}$ formaldehyde. RNA was partially hydrolyzed on gel with $5 x$ gel volumes of $0.05 \mathrm{~N} \mathrm{NaOH}$ for $20 \mathrm{~min}$. Gel was washed twice for 20 min. with 20X SSC, transferred overnight by capillarity to a HyBond-NX membrane (\#RPN303, GE Healthcare) and UV-crosslinked for fixation. For high

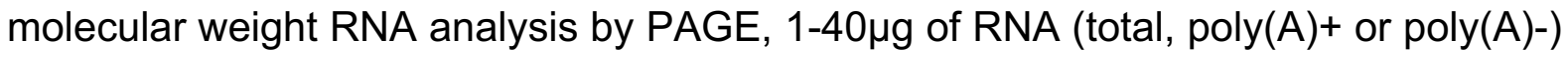
were separated on a denaturing $4 \%$ polyacrylamide-urea gel, transferred to a HyBondNX membrane by electroblotting and UV-crosslinked. For low molecular weight RNA analysis, $10-40 \mu \mathrm{g}$ of total RNA was separated on a denaturing $17.5 \%$ polyacrylamideurea gel, transferred to a HyBond-NX membrane by electroblotting and chemically crosslinked (Pall and Hamilton 2008). Probes from PCR products were radiolabeled 
using the Prime-a-Gene kit (\#U1100, Promega) in the presence of $\left[\alpha^{32} \mathrm{P}\right]-\mathrm{dCTP}$ 1096 (Hartmann Analytic) and oligo probes were radiolabeled by incubation of PNK 1097 (\#EK0031, Thermo) in the presence of $\left[\gamma^{32} \mathrm{P}\right]-\mathrm{ATP}$. Membranes were hybridized with 1098 these probes in PerfectHyb hybridization buffer (\#H7033, Sigma) and detected on a 1099 Typhoon FLA 9500 (GE Healthcare) laser scanner. Oligonucleotides used for probe generation are listed in (Supplementary table 1).

1101

1102 Proteins were separated on SDS-polyacrylamide gels, transferred to Immobilon-P 1103 PVDF membranes (\#IPVH00010, Millipore) by electroblotting and incubated with 1104 antibodies in 1X PBS with $0.1 \%$ Tween-20 and 5\% nonfat dried milk. After incubation with HRP-conjugated secondary goat antibody against rabbit or rat primary antibodies (Sigma), detection was performed with the Clarity Max Western ECL substrate (\#1705062, BIO-RAD) on a ChemiDoc Touch imaging system (BIO-RAD). Affinity1108 purified antibodies were used at the specified dilutions: GAG (1:2'000 (Oberlin et al. 1109 2017)), GFP (1:5’000 Chromotek \#3H9-100), GUS (1:1'000 Sigma Aldrich \#G5545), H3 (1:10000 Abcam \#ab1791), UGPase (1:2000 Agrisera \#AS5 086). Protein loading was confirmed by Coomassie staining of membranes.

\section{Quantitative PCR}

1114 RNA was treated with DNasel (\#EN0521, Thermo Scientific) and cDNA was 1115 subsequently synthesized with the Maxima First-Strand cDNA Synthesis Kit (\#K1641, 1116 Thermo Scientific), or RevertAid cDNA Synthesis Kit with Oligo(dT) (\#K1612, Thermo 1117 Scientific). qPCRs were run on a LightCycler480 II (Roche) or a QuantStudio5 (Applied 1118 Biosystems) machine with the SYBR FAST qPCR Kit (KAPA Biosystems). Ct values 1119 were determined by the 2nd derivative max method of minimally two technical 
replicates for each biological replicate. Relative expression values were computed as

1121 ratios of $\mathrm{Ct}$ values between targets of interest and ACT2 and/or GAPC reference

1122 mRNA unless otherwise indicated. EVD copy numbers were determined by direct

1123 qPCR on genomic DNA, comparing relative EVD and ACT2 levels, normalized by their

1124 inherent copy numbers of two and one in WT plants, respectively. Oligonucleotides

1125 used are listed in Supplementary table 1.

1126

\section{Separation of polyadenylated mRNA}

1128 Isolation of poly $(A)+$ from non-poly(A) RNA was performed from 75 ug of Trizol1129 extracted total RNA from floral buds, using the DynabeadsTM mRNA Purification Kit 1130 (Ambion Cat\#.61006) following the manufacturer's instructions. Non-polyA RNA was 1131 precipitated from the DynabeadsTM-unbound fraction and resuspended in the same 1132 volume (200 uL) as the poly(A)+ RNA fraction. Efficiency of the separation was 1133 confirmed by running aliquots of each fraction on a $1 \%$ agarose gel to monitor efficient 1134 depletion of rRNA in poly $(\mathrm{A})+$ fractions before downstream analysis.

\section{Cloning and mapping of 5'OH-ends:}

1137 Non-canonical cleavage sites in EVADE transcript were mapped by a modified

1138 5'RACE method. Total RNA isolated from a pool of 2-3 weeks old plants extracted by 1139 standard protocols (See nucleic acid extraction section) were taken for RNA ligations 1140 after DNase I treatment ((\#EN0521, Thermo scientific). RNA adapters with a 11415 ' inverted dT modification (See Supplementary Table 1) were ligated to the DNase1142 treated RNA by T4 RNA ligase 1 (\#M0204S, New England Biolabs) to render the 1143 canonical cleavage products not available for subsequent ligation reaction. To map 1144 the cleavage products with a 5' hydroxyl group, the RNA was subsequently ligated to 
1145 an RNA adapter with a 3' phosphate group by RtcB ligase (\#M0458S, New England

1146 Biolabs). The ligated RNA was converted to cDNA with RevertAid first strand cDNA

1147 synthesis kit (\#K1612, Thermo scientific) and a primer specific to the EVADE transcript

1148 (Supplementary Table 1). The cDNA was amplified by nested PCR by using primers

1149 from the adapter RNA and primers located $\sim 100$ nucleotides downstream of each

1150 stalling site (all adaptor and primers sequences can be found in Suppl. Table 1). The

1151 PCR products were separated on an agarose gel and the DNA fragments were 1152 extracted from the gel by GeneJET gel extraction kit (\#K0691, ThermoFisher 1153 scientific). The DNA fragments were cloned in pJET1.2 vectors by using CloneJET 1154 PCR cloning kit (\#K1232, ThermoFisher scientific) and 50 colonies were screened 1155 for each potential cleavage site by Sanger sequencing technology.

1156

\section{Small RNA sequencing}

1158 Small RNA sequencing of 35S:EVDwt and 35S:GFP-EVD int/ter-GUS was performed as 1159 follows. Total RNA was resolved on a $17.5 \%$ polyacrylamide-urea gel and sizes 1160 between 18 - 30 nt were excised, eluted overnight in elution buffer (20mM Tris-HCl 1161 (pH 7.9), 1 mM EDTA, 400 mM ammonium acetate, 0.5\% (w/v) SDS) and collected by 1162 precipitation with equal volumes of isopropanol. RNA was quantified using the Qubit ${ }^{\mathrm{TM}}$ 1163 RNA HS Assay Kit (Thermo Scientific) and subsequently cloned using the Small RNA1164 Seq Library Prep Kit (Lexogen). Sequencing was performed on an Illumina HiSeq 11654000 machine.

1166

\section{RIBO-seq}

1168 For RIBO-seq libraries frozen inflorescence tissue was ground in digestion buffer (100 $1169 \mathrm{mM}$ Tris $\cdot \mathrm{HCl}(\mathrm{pH} 8), 40 \mathrm{mM} \mathrm{KCl}, 20 \mathrm{mM} \mathrm{MgCl}_{2}, 2 \%(\mathrm{v} / \mathrm{v})$ polyoxyethylene (10) tridecyl 
1170 ether, $1 \%$ (v/v) de-oxycholic acid, $1 \mathrm{mM} \mathrm{DTT}, 10$ unit/mL DNase I (Thermo Scientific),

$1171100 \mu \mathrm{g} / \mathrm{mL}$ cycloheximide). Precleared solutions were incubated with $650 \mathrm{U}$ RNase I

1172 (Ambion) for $45 \mathrm{~min}$ at $25^{\circ} \mathrm{C}$. Nuclease digestion was stopped by the addition of $10 \mu \mathrm{l}$

1173 SUPERase In RNase Inhibitor (Ambion). Resulting monosomes were purified by 1174 ultracentrifugation of the lysate on a sucrose cushion (1 M sucrose, $20 \mathrm{mM}$ HEPES 1175 (pH 7.6), 100 mM KCl, 5 mM MgCl2, $10 \mu \mathrm{g} / \mathrm{ml}$ cycloheximide, 10 units/ml RiboLock 1176 (Thermo Scientific) and cOmplete protease inhibitor cocktail (Roche) for 4 hours at 1177250 '000 $\mathrm{g}$ in $4^{\circ} \mathrm{C}$. RNA was extracted using the TRIzol RNA extraction described 1178 above and treated with $10 \mathrm{U}$ PNK (Thermo Scientific) for $30 \mathrm{~min}$. Ribosomal RNA 1179 depletion was performed using the RiboMinus Plant Kit (Thermo Scientific) and 1180 libraries were generated as above, except that the 25 - 32 nt RNA fraction was excised 1181 from the denaturing polyacrylamide gel prior to RNA ligation.

\section{3 nanoPARE}

1184 NanoPARE library preparation and analysis was performed following the protocol from 1185 Schon et al. 2018 (Schon et al, 2018). Briefly, 10ng of total RNA was isolated from inflorescences. Two biological replicates each of Col-0 and ddm1-2 were used for

1187 reverse transcription. After 9 cycles of PCR pre-amplification, 5 ng aliquots of cDNA 1188 were separately tagmented and amplified using either standard Smart-seq2 Tn5 1189 primers or 5'-end enrichment primers. The resulting Smart-seq2 and nanoPARE 1190 libraries were sequenced on an Illumina HiSeq 2500 using paired-end 50bp reads and 1191 single-end 50bp reads, respectively. 
1194 Plants germinated and grown in parallel under the same conditions were individually

1195 covered with paper bags before the maturation of siliques and harvested upon

1196 ripening. Total amount of seeds from each plant was counted twice with a C3 High

1197 Sensitive Seed Counter (Elmor).

1198

1199 Data Analysis

1200 Analysis of sRNA sequencing is based on the following workflow. Reads were trimmed 1201 using bbduk (BBTools: sourceforge.net/projects/bbmap/, version 38.41; ktrim=r k=23 1202 mink=11 hdist=1) mapped against the TAIR10 Arabidopsis genome with STAR (Dobin 1203 et al, 2013) (version 2.5.2a; --outFilterMismatchNoverLmax 0.05 --outFilterMatchNmin 120416 --outFilterScoreMinOverLread 0 --alignIntronMax 500 --alignIntronMin 50 -1205 outFilterMultimapNmax 50), quantified using Rsubread (Liao et al, 2013) (version 1206 1.20.6; allowMultiOverlap=T,largestOverlap= T, isPairedEnd=F, strandSpecific=1, 1207 countMultiMappingReads=T, fraction $=\mathrm{T}$ ) and differential analysis using 1208 DESeq2(Love et al, 2014) (version 1.10.1). Reads were split in different lengths with 1209 Samtools (Li et al, 2009) (version 0.1.19) and locus coverage amongst those read 1210 length was visualized using BEDtools (Quinlan \& Hall, 2010) (version 2.15.0 ) and R 1211 cran (version 3.2.5).

1212

1213 RIBO-seq libraries were analyzed as follows. Reads were trimmed of adapter 1214 sequences with bbduk as above. Reads mapping to rRNA loci using Bowtie2 1215 (Langmead \& Salzberg, 2012) (version 2.2.1; -k 1 -x) were discarded from further 1216 analysis. Subsequent mapping and quantification were performed as for the sRNA 1217 sequencing analysis using STAR (Dobin et al, 2013) and Rsubread (Liao et al, 2013) 1218 as above, but reads were mapped to both Arabidopsis genome and transcriptome 
1219

1220

\section{1}

1222

1223

1224

1225

1226

1227

1228

1229

1230

1231

1232

1233

1234

1235

1236

1237

1238

1239

1240

1241

1242

1243

sequences. Quality control of the RIBO-seq libraries was performed with the riboWaltz (Lauria et al, 2018) (version 1.1.0) package. P-site occupancies were estimated using the RiboProfiling (Popa et al, 2016) (version 1.0.3) package based on 5' read offsets determined by the coverage profile around start codons dependent on read lengths. Codon occupancies were compiled for all three possible frames to generate a single codon occupancy score. A ribosomal stalling score at each codon position was defined as the ratio of observed over expected counts, where the expectation was the mean of occupancy counts over the entire transcript. To improve quality of the assessment, only the most translated isoform per gene and only isoforms with a minimal read coverage of $70 \%$ were considered. Codon dwell time was estimated as the mean value of log-normalized codon occupancies per individual transcript and codon usage was estimated from the subset of genes considered translated. Stop codons and stop codons containing di-codons were excluded from the analysis. Data were visualized using R cran and the packages Gviz (Hahne \& Ivanek, 2016) and ggplot2 (Wickham).

\section{Data Access}

Sequencing data generated in this study are accessible on the Gene Expression Omnibus (GEO) under the accession number GSE167484. Data from previous studies including sRNA sequencing in $d d m 1 \& d d m 1 r d r 6, d d m 1 \& d d m 1 d c / 1$, isoform specific sequencing data of total and polysome associated mRNA in TE de-repressed backgrounds are found under the accession numbers GSE41755 (Nuthikattu et al, 2013), GSE52952 (Creasey et al, 2014), GSE93584 (Oberlin et al, 2017) and PRJNA598331 (Kim et al, 2021). Every other raw data used in this study (including raw image files, qPCR data, Sanger sequencing traces,...) has been deposited in Zenodo (www.zenodo.org) under the DOI: 10.5281/zenodo.4751668. 


\section{Figure 1}

A

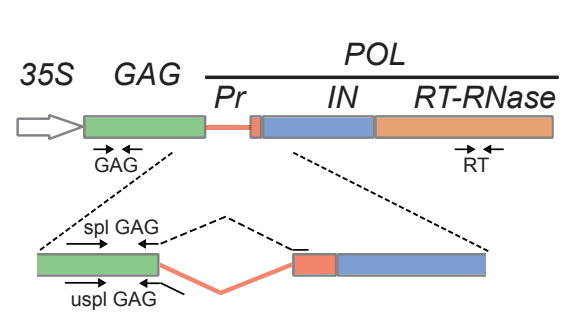

GAG-POL (unspliced) shGAG (spliced) $\triangleleft$ 1 (A)
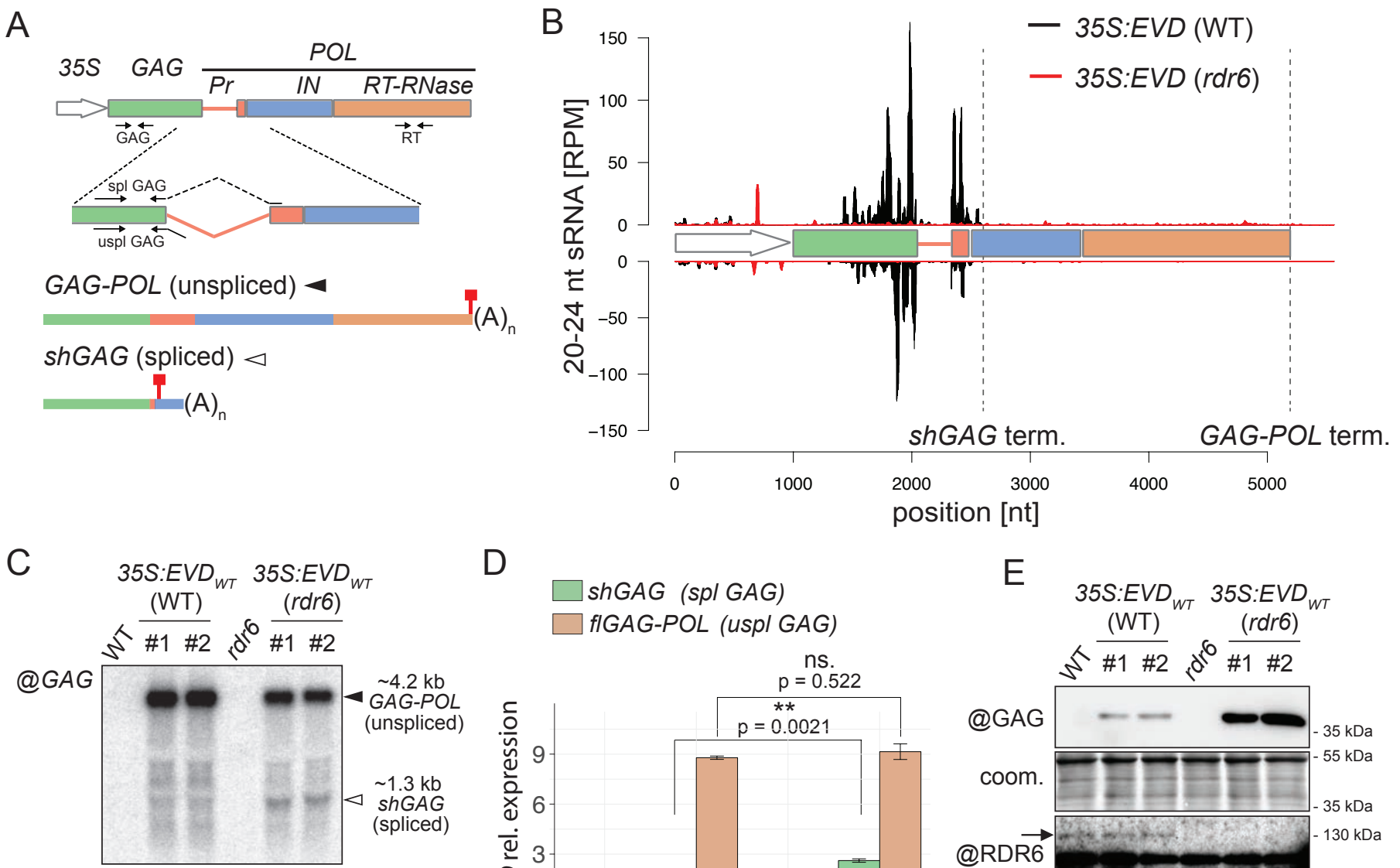

@ACT2

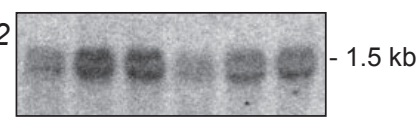

$\mathrm{D}$

$\operatorname{shGAG~(spl~GAG)~}$

fIGAG-POL (uspl GAG)
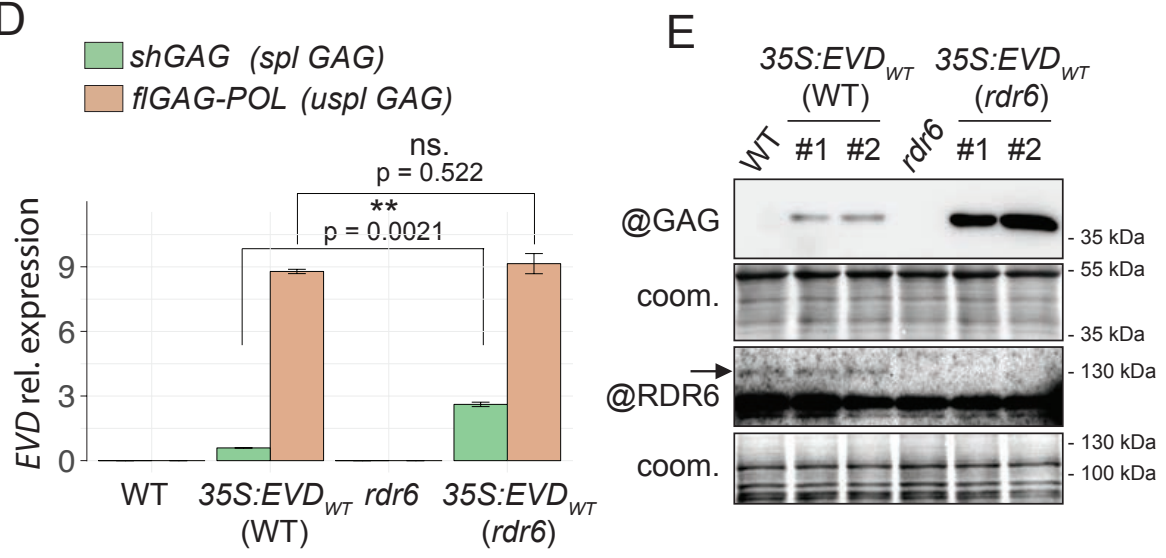

$\mathrm{F}$

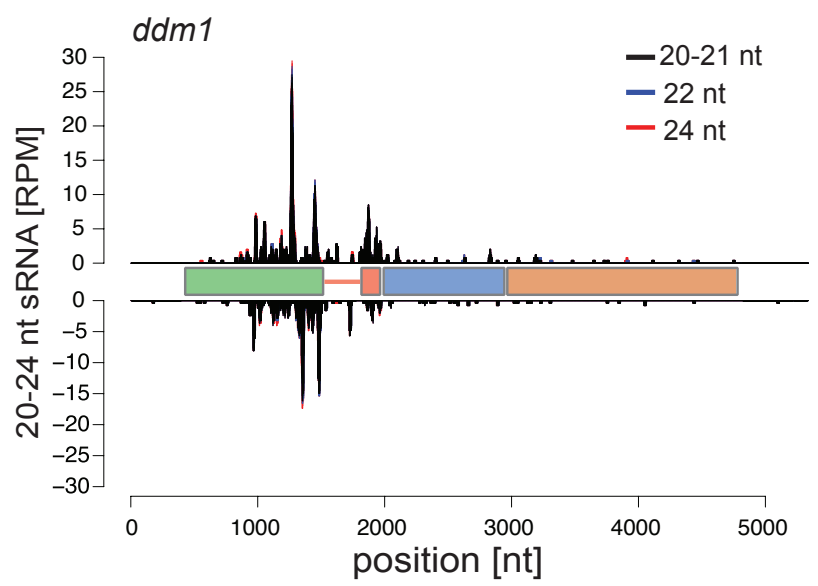

G

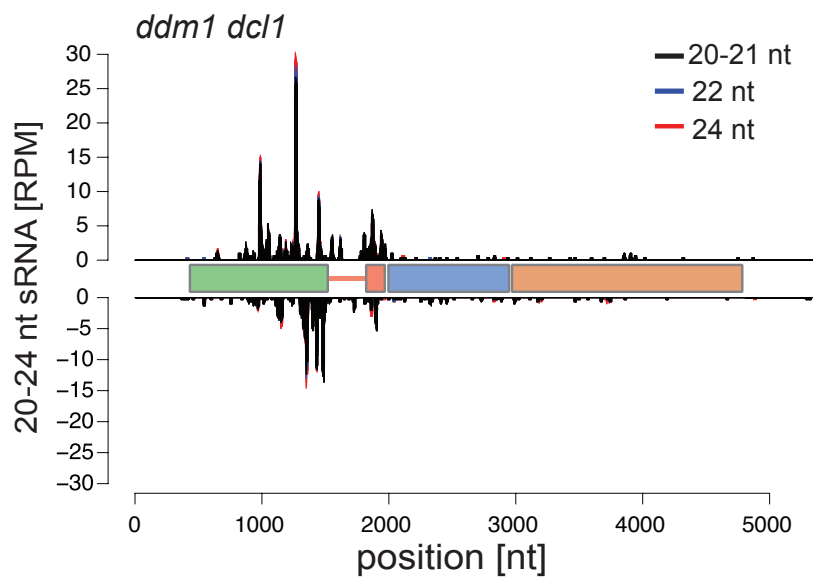

Figure 1. EVD shGAG is both a trigger and a target of RDR6-dependent but miRNA-independent siRNAs.

(A) EVD flGAG-POL and spliced shGAG mRNAs are distinguishable using specific PCR primer sets (arrows) for quantification and northern analysis. (35S) Cauliflower Mosaic Virus 355 promoter, (Pr) protease, (IN) integrase, (RT-RNase) reversetranscriptase-RNase; red squares: stop codons. (B) sRNA-seq reads profile of EVD expressed from 35S:EVDWT in WT (black) or rdr6 (red). (RPM) reads per million. Positions are indicated in nucleotides (nt) from the start of the 355 sequence. Dashed vertical lines: shGAG and GAG-POL 3' ends. (C) Northern analysis of EVD RNA isoforms using a probe for the GAG region or for ACTIN2 (ACT2) as a loading control. (D) qPCR quantification of shGAG and fIGAG-POL normalized to ACT2 and to GLYCERALDEHYDE-3-PHOSPHATE DEHYDROGENASE C SUBUNIT (GAPC) levels. qPCR was performed on $\mathrm{n}=3$ biological replicates; bars: standard error. $\left({ }^{* *}\right)=p$-value $<0.01$ (two-sided t-test between indicated values). (E) Western analysis of GAG and RDR6 with Coomassie (coom.) staining as a loading control. (F-G) sRNA-seq profiles from EVD de-repressed in the $d d m 1$ (F) or $d d m 1 d c 11$ (G) backgrounds. Different siRNA size categories are stacked. Nomenclature as in (B). 


\section{Figure 2}

A

(EVD)

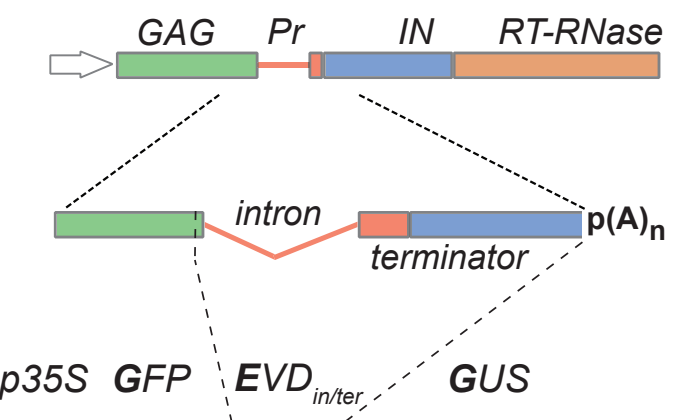

(GEG)

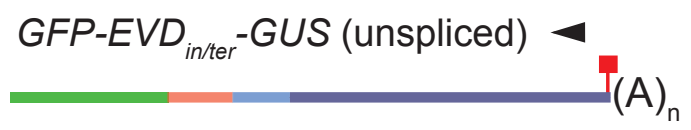

$\operatorname{shGFP}($ spliced) $\triangleleft$

$(\mathrm{A})_{\mathrm{n}}$
B
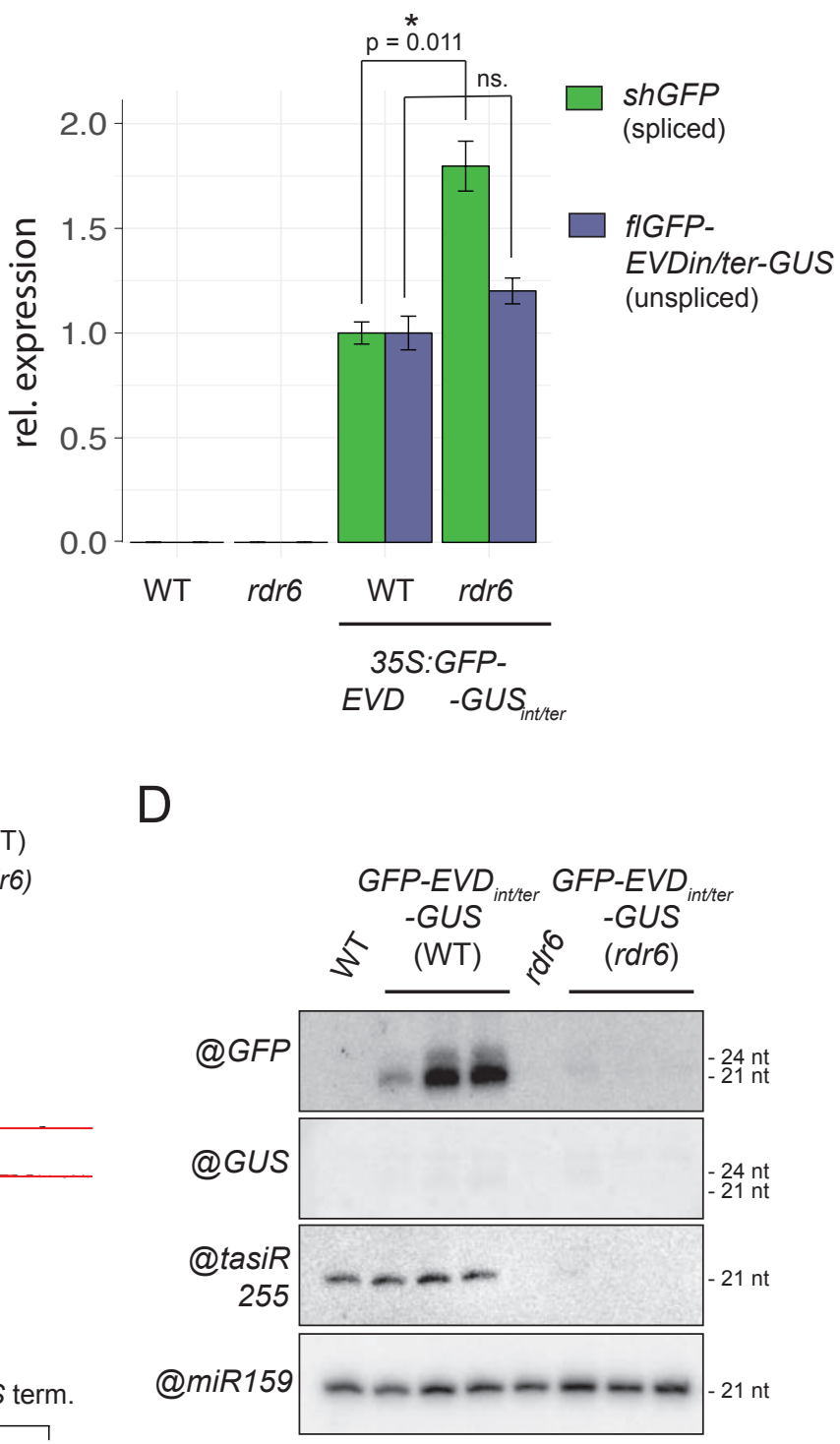

$\mathrm{D}$

C

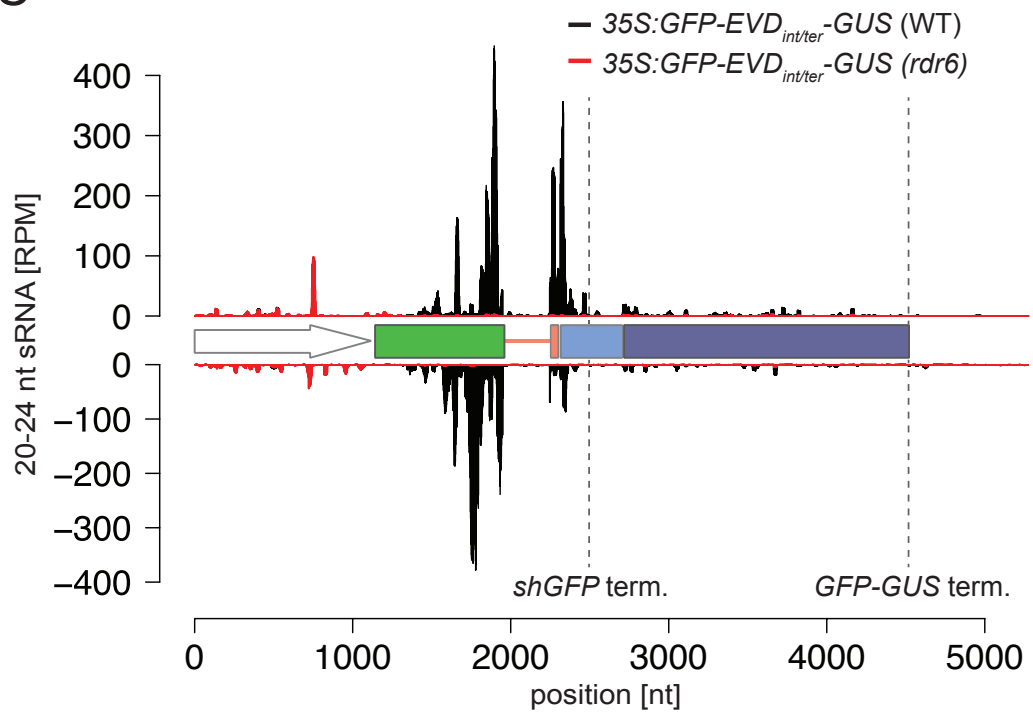

\section{Figure 2. The EVD intron and terminator suffice to initiate PTGS.}

(A) The 35S:GFP-EVDint/ter-GUS fusion was made by introducing the EVD intron and proximal shGAG terminator between the GFP and GUS coding sequence. Like EVD, it spawns full length unspliced and short spliced mRNAs. Red squares: stop codons. (B) Expression levels of shGFP (spliced) and GFP-EVDint/ter-GUS (unspliced) transcripts, relative to ACT2 and AT4G26410 (RHIP1), in the WT or rdr6 background. qPCR was performed on three biological replicates and error bars represent the standard error on. $\left(^{*}\right)=p$-value $<0.05$ (two-sided t-test against corresponding controls). (C) sRNA-seq profile mapped on the genomic 35S:GFP-EVDint/ter-GUS locus. (RPM) Reads per million. Positions indicated in nucleotides (nt) from the start of the $35 \mathrm{~S}$ sequence. Dashed vertical lines: shGFP and GFP-GUS 3' ends. (D) Low molecular weight RNA analysis of the GFP- and GUS-spanning regions. tasiRNA255 is a control for the rdr6 mutation and miR159 provides a loading control. 


\section{Figure 3}

A

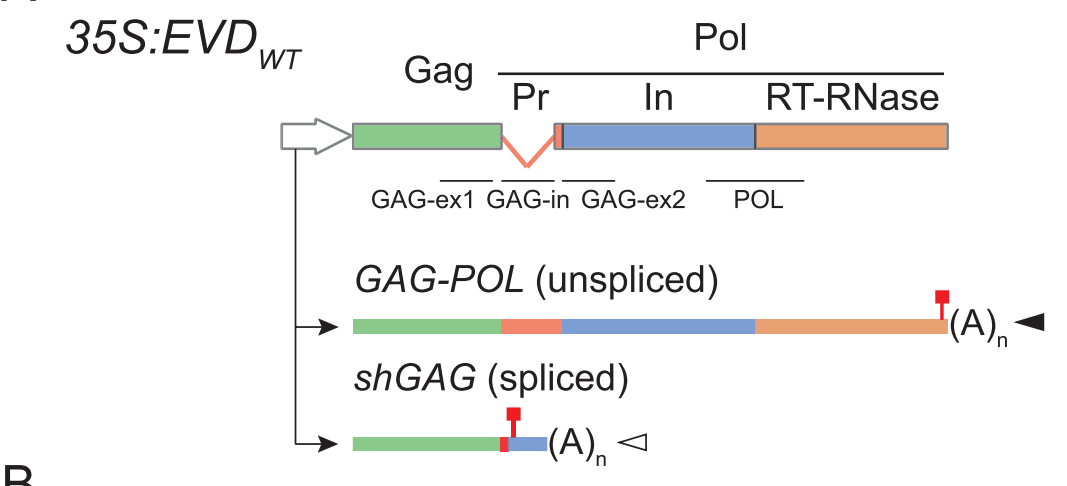

B

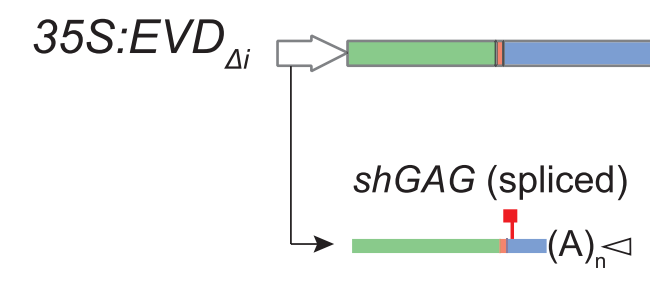

C

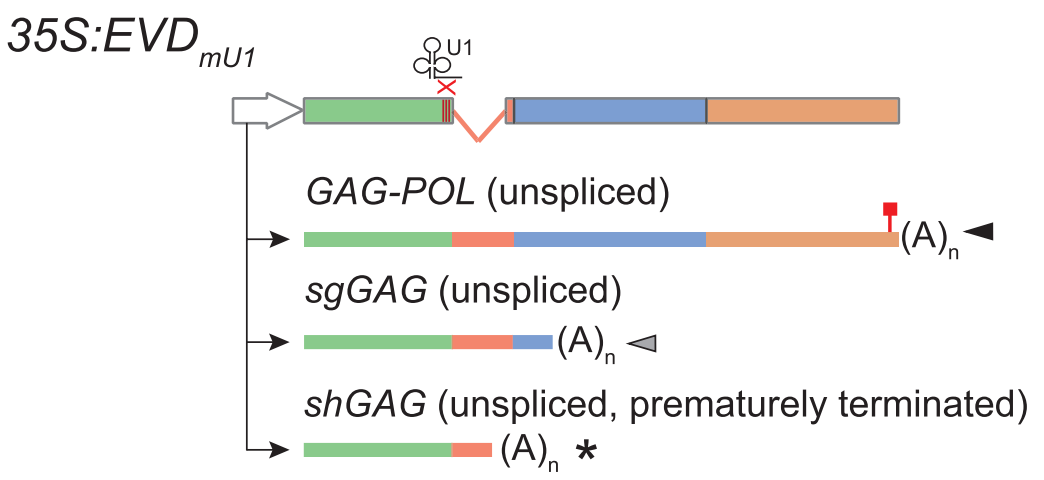

D

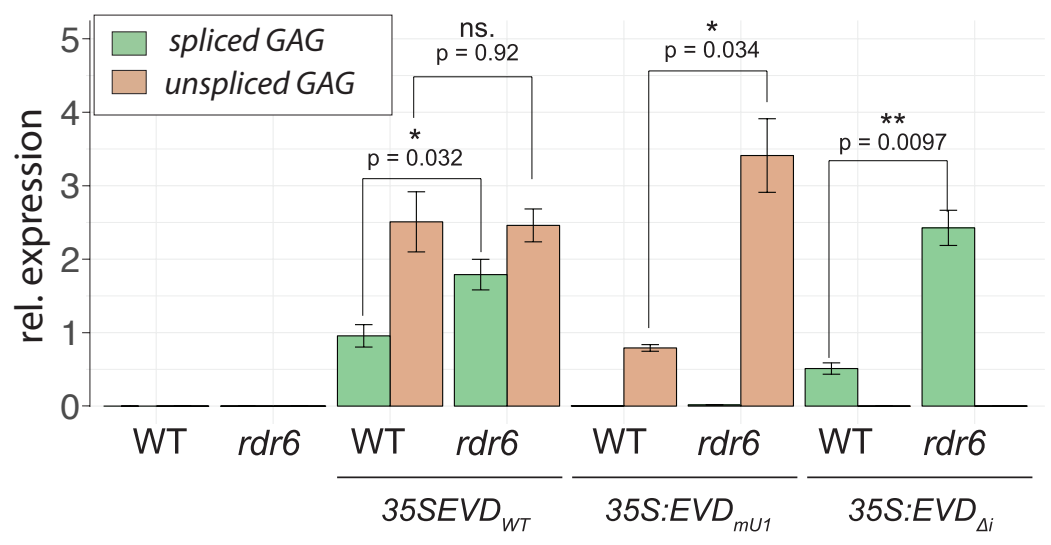

E

35S:EVD ${ }_{W T} 35 S: E V D_{m U 1} 35 S: E V D_{\Delta i}$
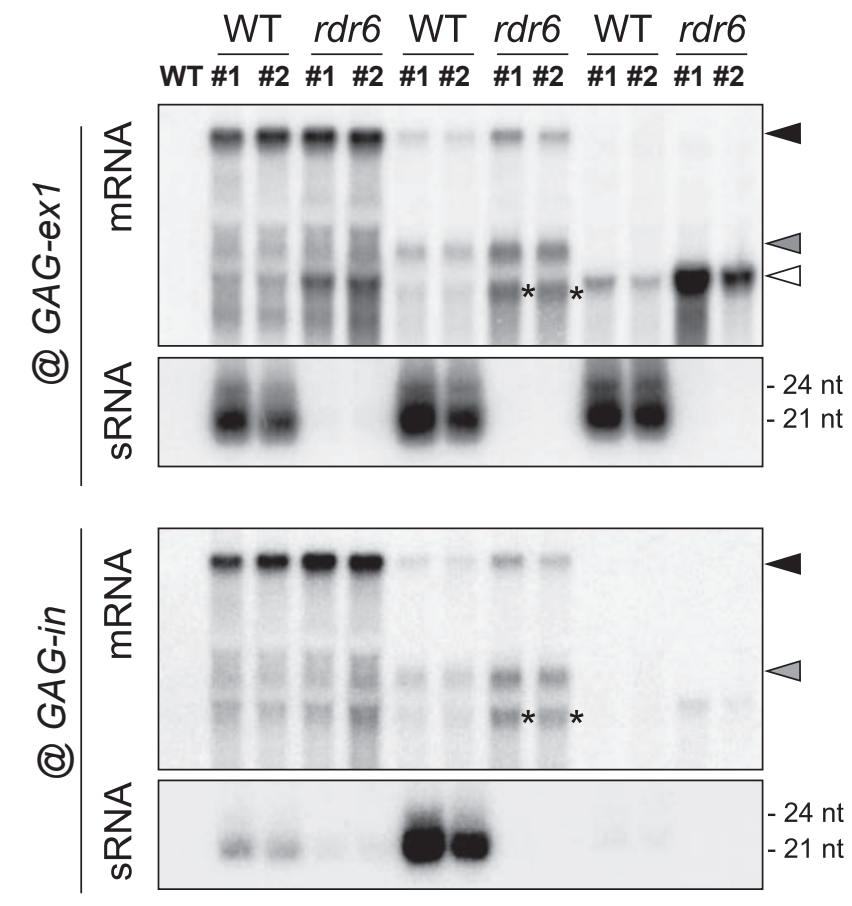

$@ A C T 2$
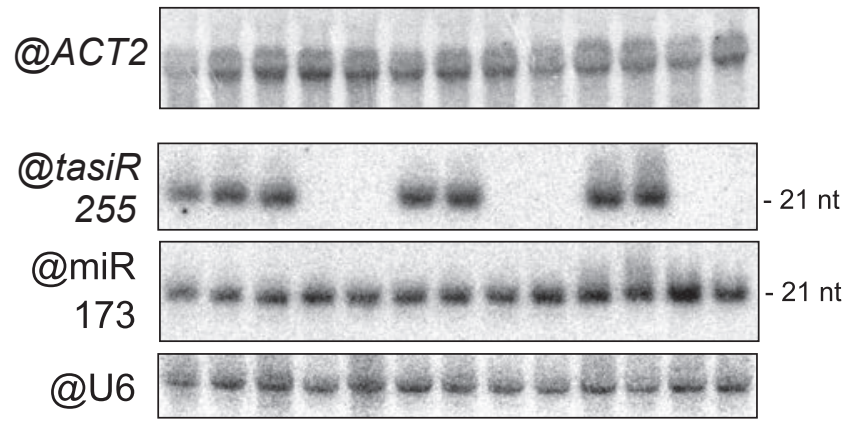

$\mathrm{F}$ 35S:EVD ${ }_{W T} 35 S: E V D_{m U 1} 35 S: E V D_{\Delta i}$ WT $r d r 6$ WT $r d r 6$ WT $r d r 6$

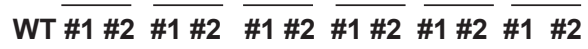

@ GAG

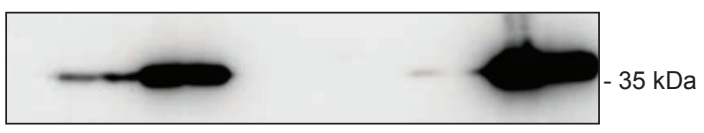

coom.

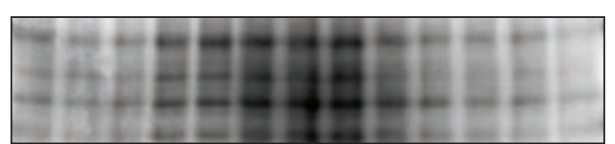

Figure 3. Impact of splicing and premature termination on EVD silencing.

(A-C) Constructs and isoforms transcribed from 35S:EVDwt (A), 35S:EVDAintron (B) and 35S:EVDmU1 (C). Probes for northern analysis of GAG exon 1 (GAG-ex1), intron (GAG-in), exon2 (GAG-ex2) and the POL region are depicted with black lines. (D) Relative expression levels of spliced and unspliced transcripts in the three EVD constructs relative to ACT2. qPCR was performed on three biological replicates and error bars represent the standard error. (ns.) = nonsignificant, $\left(^{*}\right)=p$-value $\left.<0.05,{ }^{* *}\right)=p$-value $<0.01$, (two-sided t-test between indicated samples/targets). (E) High and low molecular-weight RNA analysis of EVD GAG (GAG-ex1) and EVD intron (GAG-in) in two independent T1 bulks from each indicated line. The filled arrows on the right hand-side or with an asterisk on the blots correspond to the transcripts depicted in (A-C). ACT2: loading control for mRNAs; tasiR255, miR173 and U6: loading controls for sRNAs. Hybridizations for GAG-ex2 and POL probes are found in Supp. Fig.5A. (F) Western analysis of the GAG protein with Coomassie (coom.) staining as a loading control. 


\section{Figure 4}

A siRNA quartiles

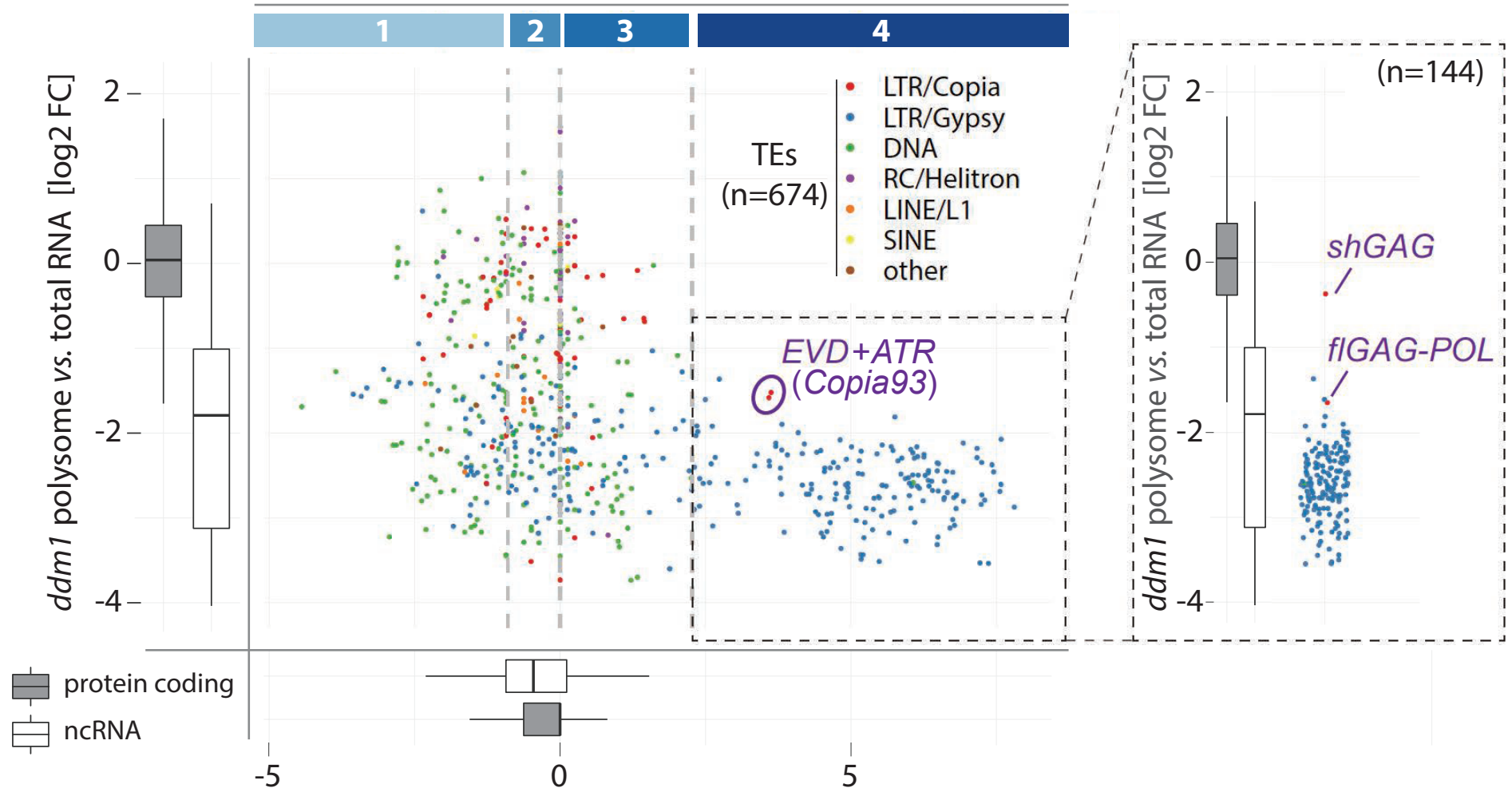

20-21 nt siRNA $d d m 1$ vs. $d d m 1$ rdr6 [log2 FC]

B
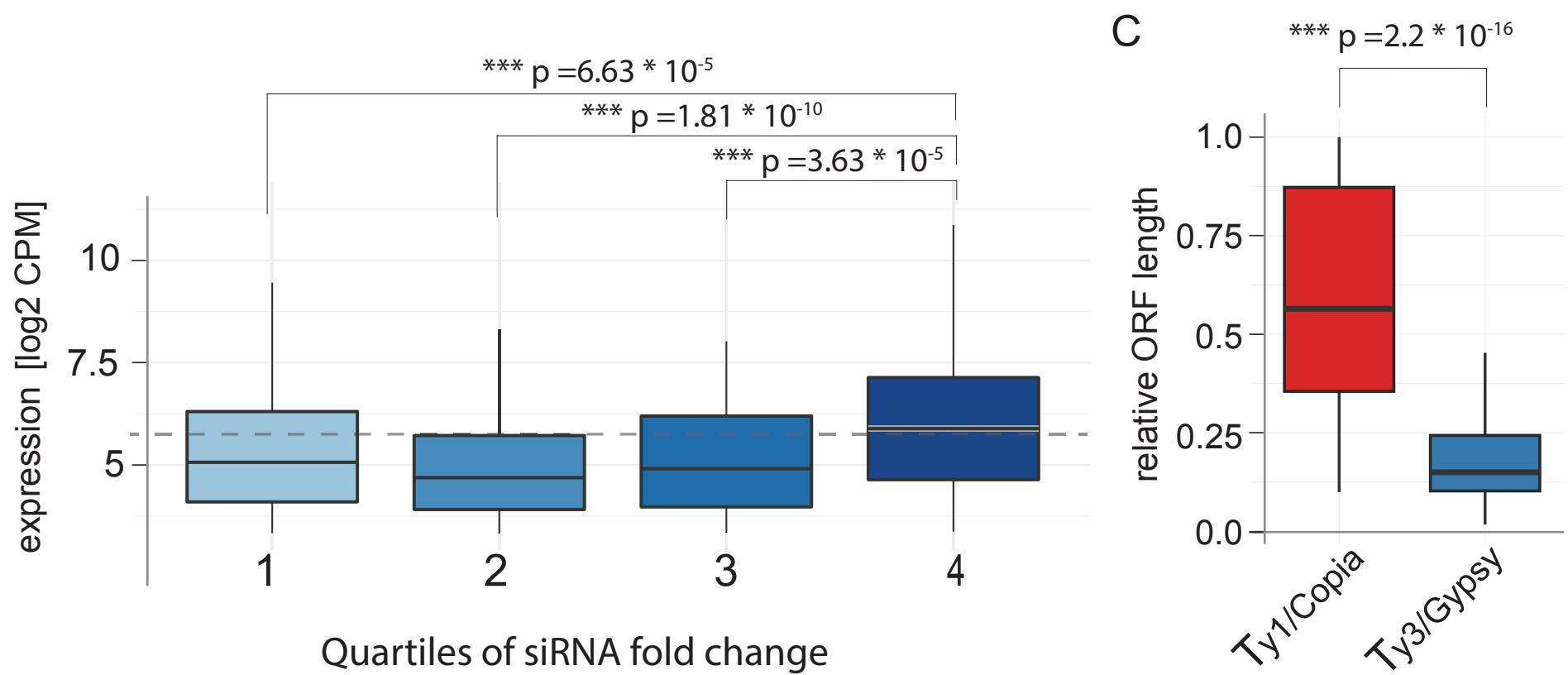

Quartiles of siRNA fold change

Figure 4. Expression, but not translation, is associated with RDR6 activity on most $d d m$ 1-reactivated TEs

except EVD. (A) Scatter plot comparing polysome association score (defined as fold-change between abundance in polysome libraries vs. total RNA) and RDR6-dependent siRNA levels of TEs found de-repressed in ddm 1 (brief description of RDR6 dependency). Quartiles of siRNA levels are confined by gray vertical lines. For comparison and reference, polysome association and RDR6-dependent siRNA levels of protein coding and non-coding transcripts are displayed as boxplots. Copia93 elements: EVD (AT5G17125) + ATR (AT1G34967), are circled. Inlet: Polysome association score of TEs in quartile 4, EVD mRNA isoforms are displayed separately. (B) Boxplots of RNA expression levels of TEs in $d d m 1$ from the quartiles in (A). In all panels: $\left.{ }^{* * *}\right)=p$-value $<0.001$, (Wilcoxon rank-sum test against labelled controls or protein coding gene cohort). (C) ORF length of Ty1/Copia and Ty3/Gypsy elements expressed in ddm 1 relative to their genomic length. 
Figure 5
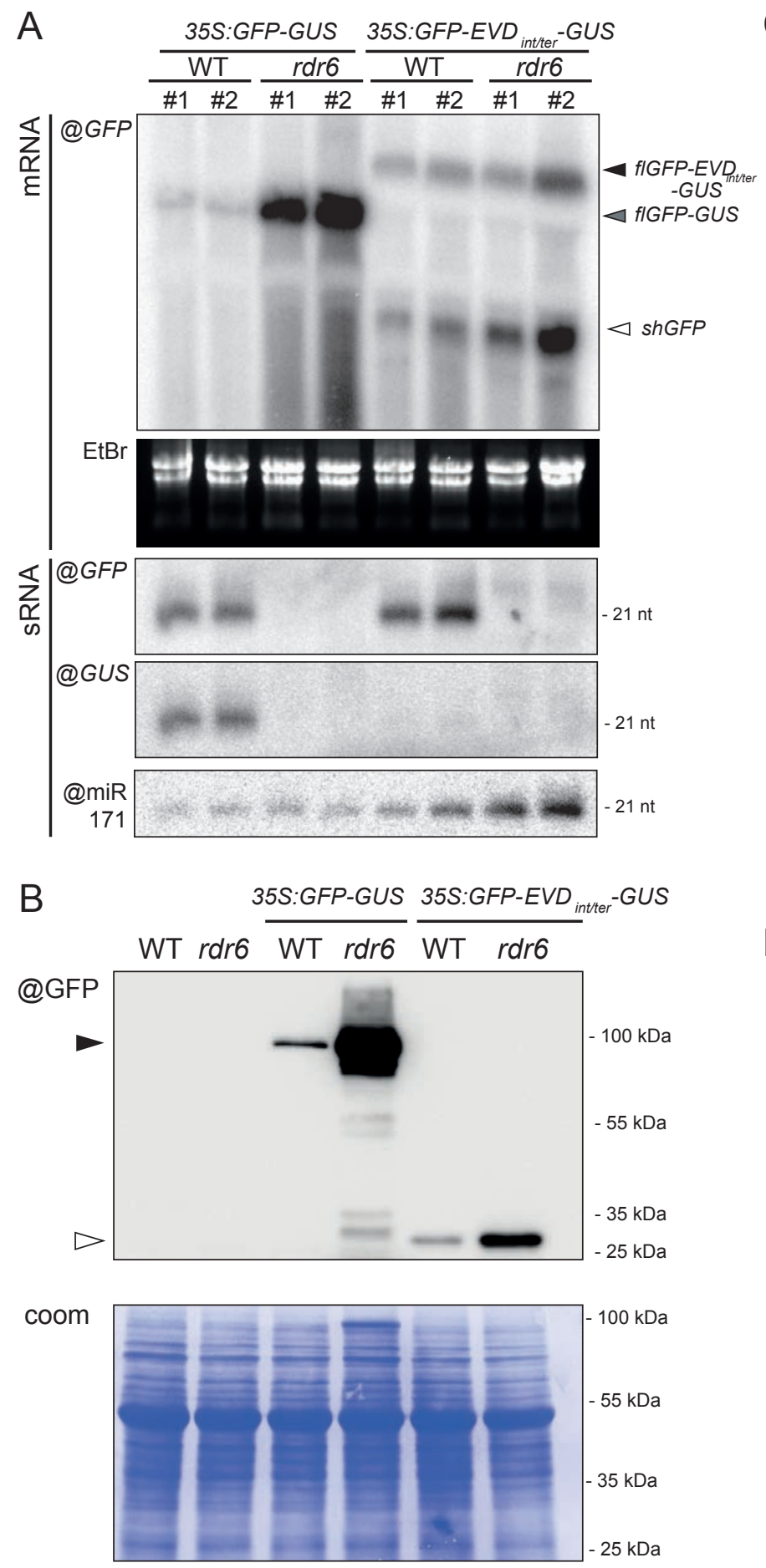
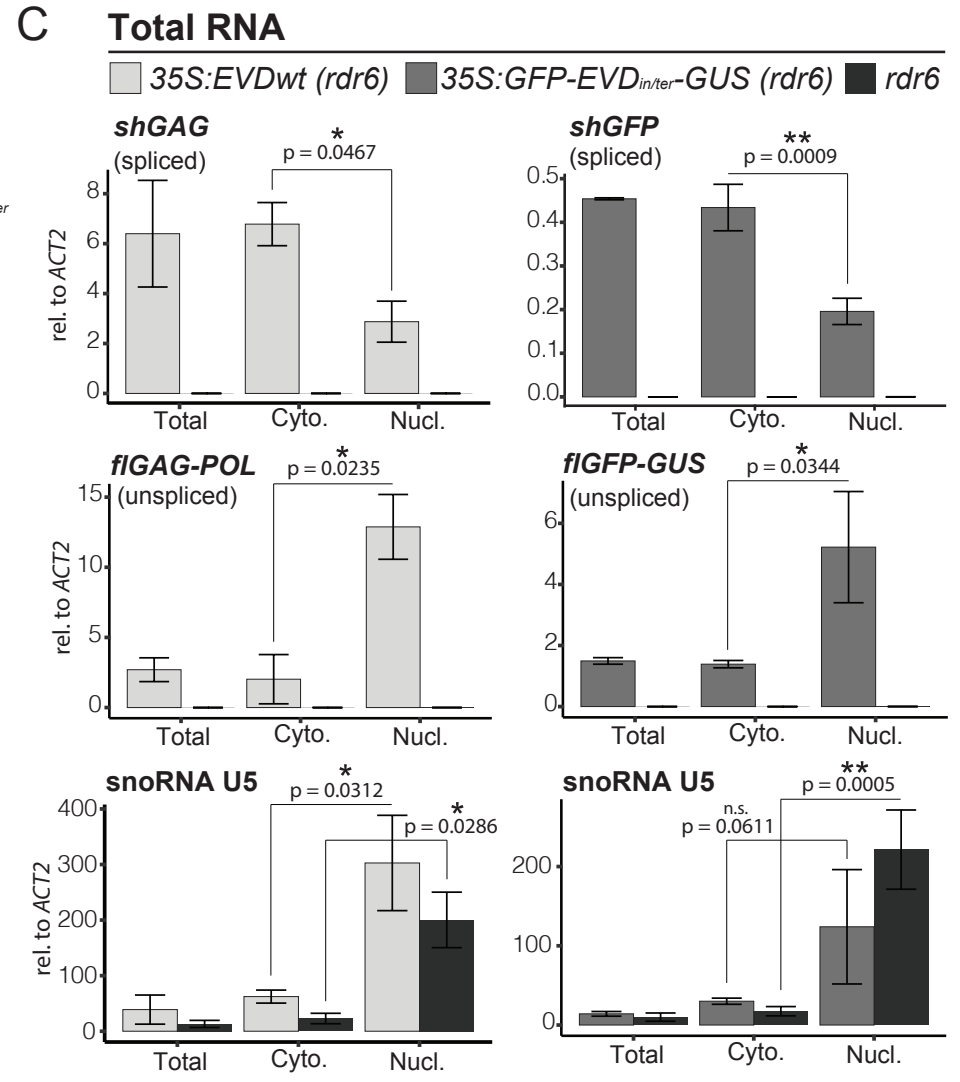

D $\operatorname{Poly}(\mathbf{A})+$ RNA

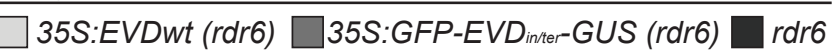
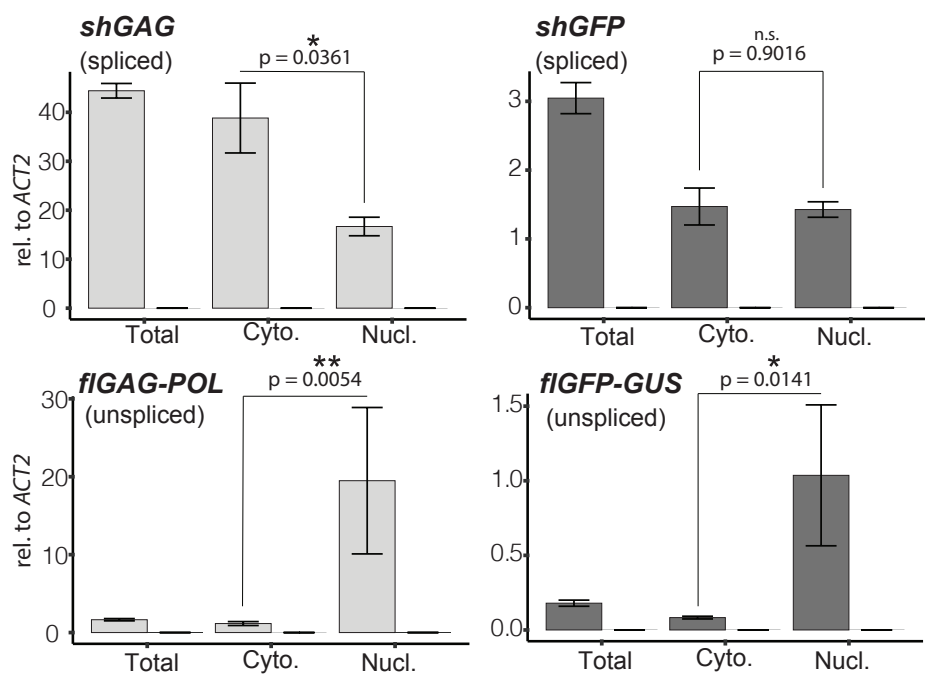

Figure 5. Splicing promotes translation and siRNA biogenesis from short-spliced mRNAs by influencing nucleocytoplasmic distribution of RNA isoforms. (A) Comparison of RNA isoforms and sRNA patterns generated by 35S:GFP-GUS and 35S:GFP-EVDint/ter-GUS. High and low molecular-weight RNA analysis using a GFP or GUS probe in two independent transgenic lines from each construct in the WT or rdr6 background. mRNA isoforms are indicated with arrows and correspond to the transcripts depicted in Fig.2A. EtBr staining of the agarose gel and miR171 probe serve as loading control for mRNAs and sRNAs, respectively. (B) Western analysis of the translation products from GFP and GFP-GUS transcripts. Coomassie (coom.) staining as a loading control. (C) Nuleo-cytosolic distribution of 35S:EVD and 35S:GFP-EVDint/ter-GUS RNA isoforms in rdr6 relative to that of ACT2 analyzed by qPCR. RNA extracted from Total, nuclear (Nucl) and cytoplasmic (Cyto) fractions was reverse transcribed with random hexamers and oligo(dT). snoRNA U5 is shown as a nuclear-only RNA control. (D) Same as in (C) but using exclusively oligo(dT) to reverse transcribe poly(A)+ RNAs. Both in (C) and (D), qPCR was performed on $n=3$ biological replicates; bars: standard error. $\left(^{*}\right)=p$-value $<0.05$, $(* *)=p$-value $<0.01$ (two-sided t-test between indicated samples). 

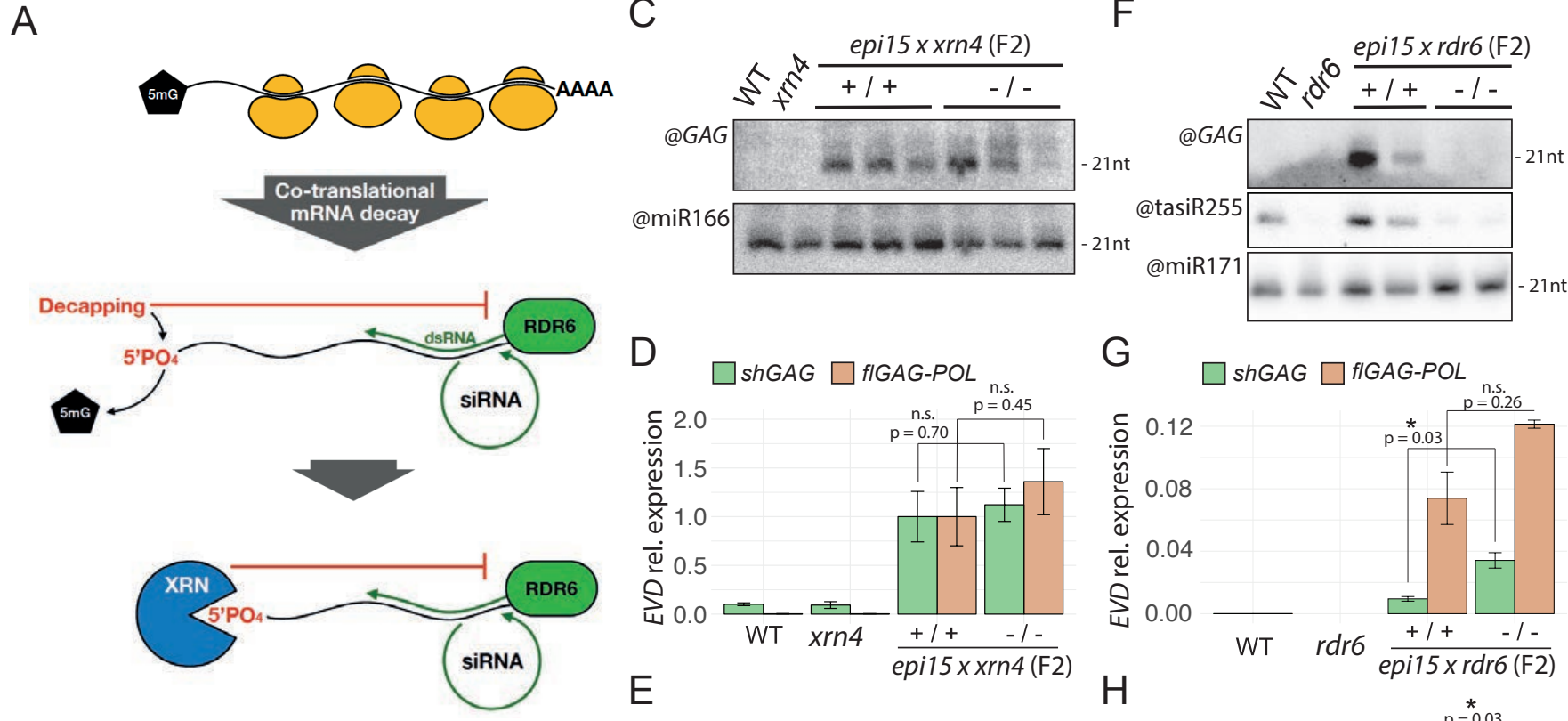

B
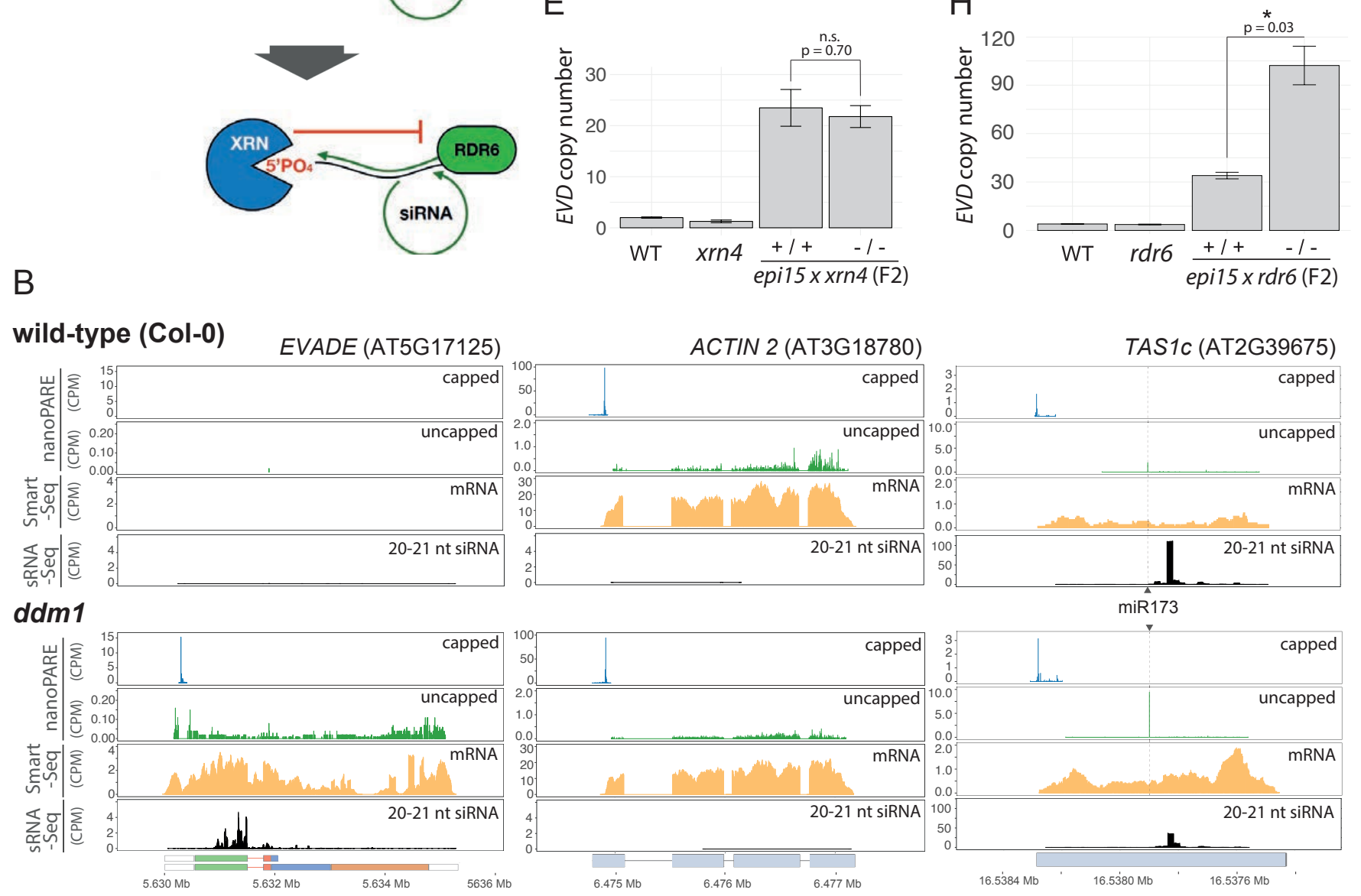

Figure 6. XRN4-mediated co-translational mRNA decay does not influence siRNA production from shGAG. (A) During co-translational decay, mRNA turnover is initiated by decapping of actively translated mRNAs. This exposes $5^{\prime}$ monophosphate (5'PO4) groups required for the 5'->3' exonucleolytic activity of XRNs. As evidenced by the RDR6-dependent production of siRNAs from dozens of endogenous loci in decapping and xrn4 mutants of Arabidopsis, plant mRNAs engaged in co-translational decay can become substrates for siRNA biogenesis if they are not degraded by, or their levels saturate, this process. While depicted here, for simplicity, as a co-translational event, RDR6 action onto such RNA likely occurs in specialized "siRNA bodies" adjacent to P-bodies (B) EVD, ACT2 and TAS1c capped and uncapped 5'end from nanoPARE and Smart-seq2 libraries along 20-21 nt siRNA in WT and ddm1. (C-E) EVD genomic proliferation in homozygous xrn4 mutant and WT backgrounds in F2 plants from a cross between xrn4 and epi 15 exhibiting active EVD mobilization. (C) sRNA blot analysis using an anti-GAG probe. (D) Relative expression levels of shGAG and flGAGPOL normalized to ACT2 and to AT4G26410 levels. (E) EVD genomic copy number quantification by qPCR. qPCR analysis was performed on three biological replicates for controls and the three independent WT and mutant F2 lines displayed in B. (F-H) Same as (C-E) but with the rdr6 versus WT epi15 backgrounds. Analysis was performed in the two independent WT and mutant F2 lines displayed in E. In all qPCR panels: error bars display standard errors. (ns.) = non-significant, $\left(^{*}\right)=p$-value $<0.05,(* *)=p$-value $<0.01$ (two-sided t-test between indicated samples) 


\section{Figure 7}

A
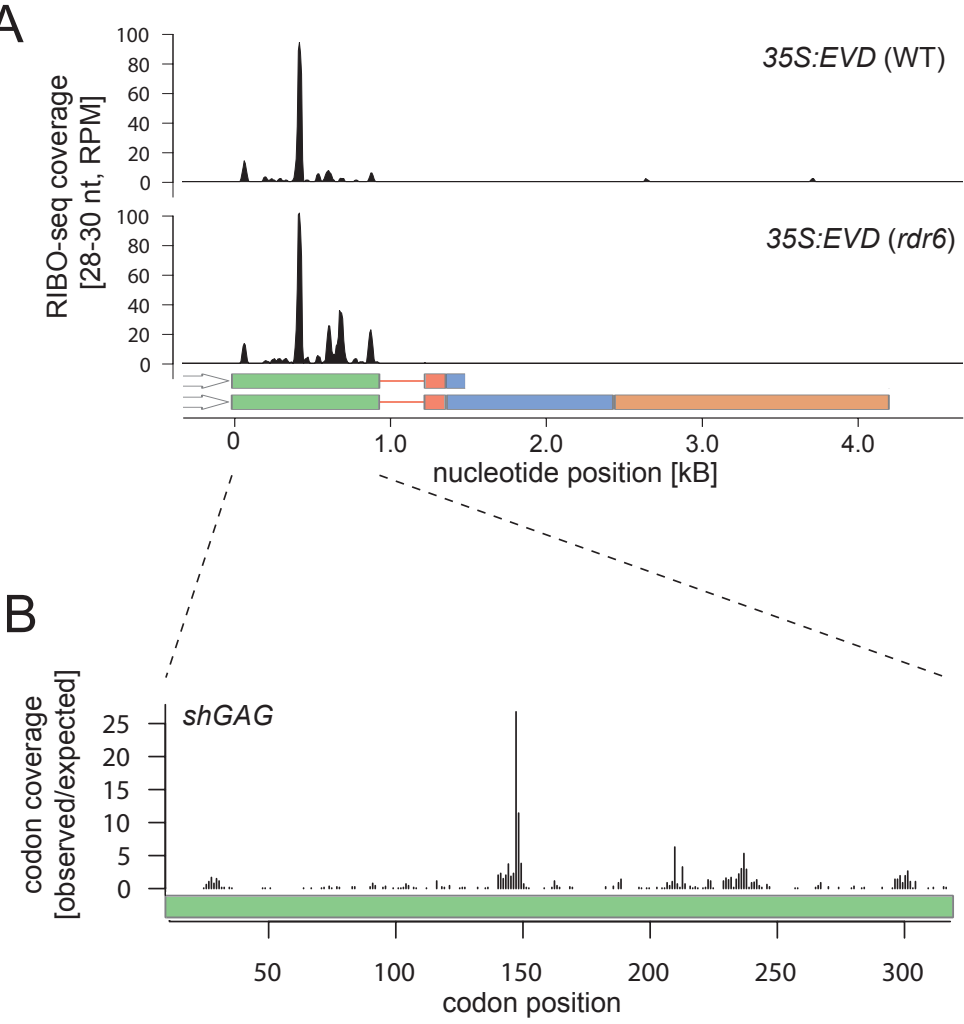

E
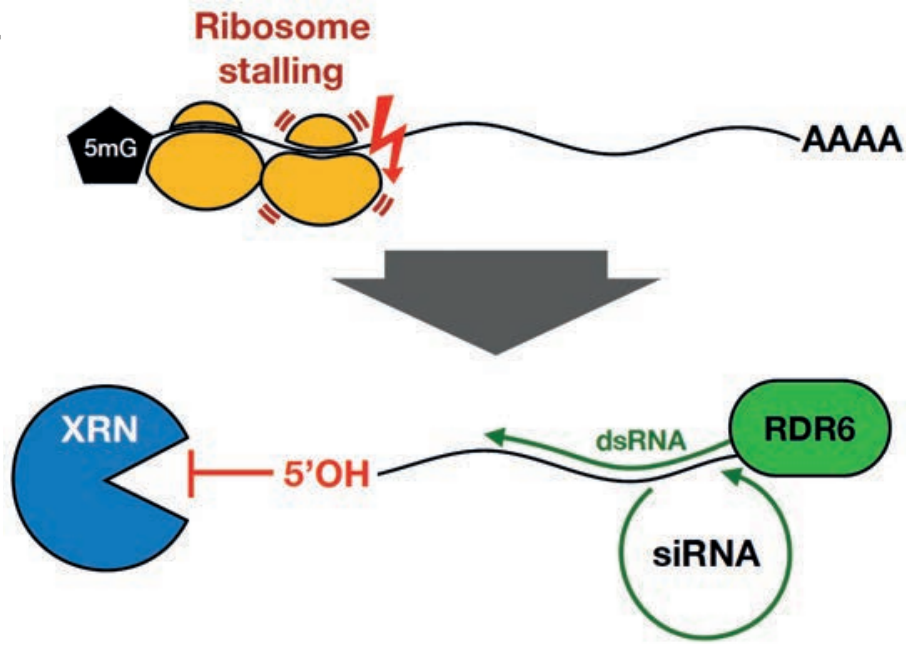

C
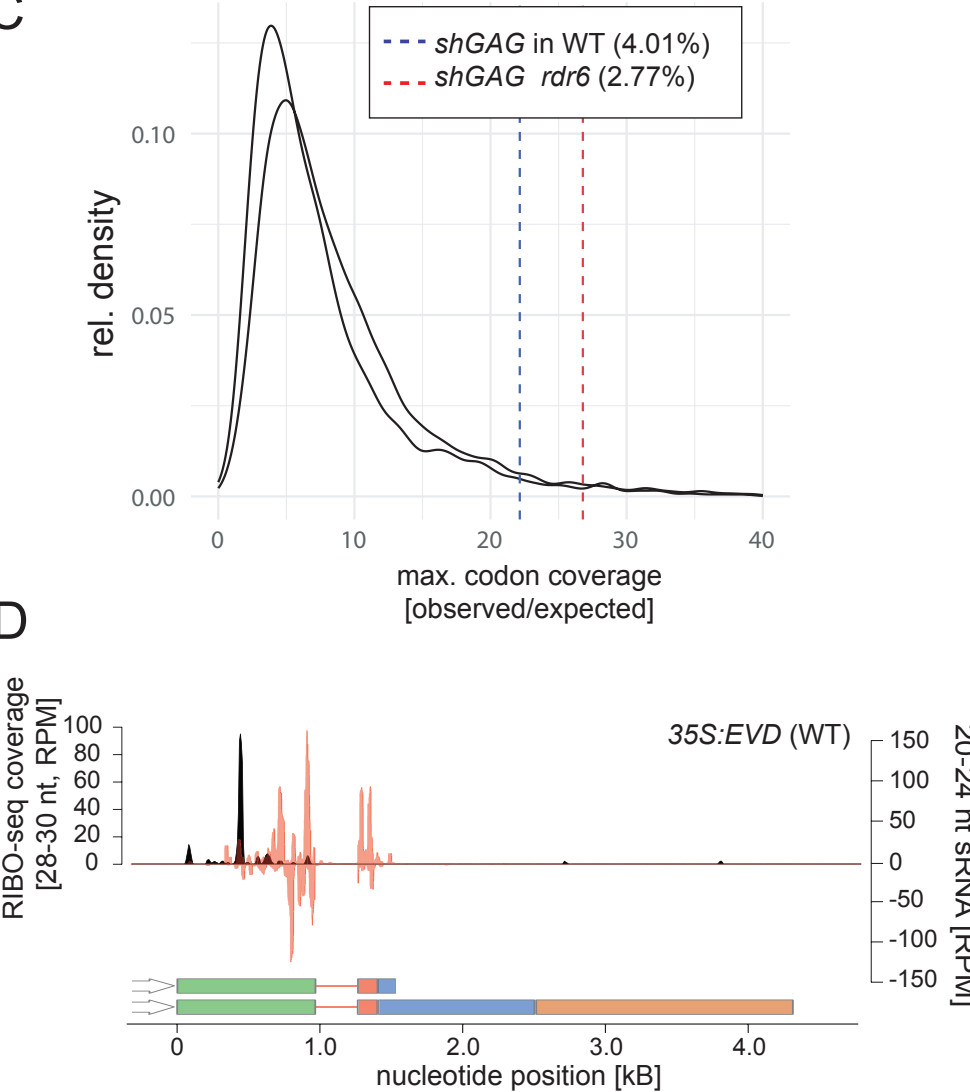

$\mathrm{F}$

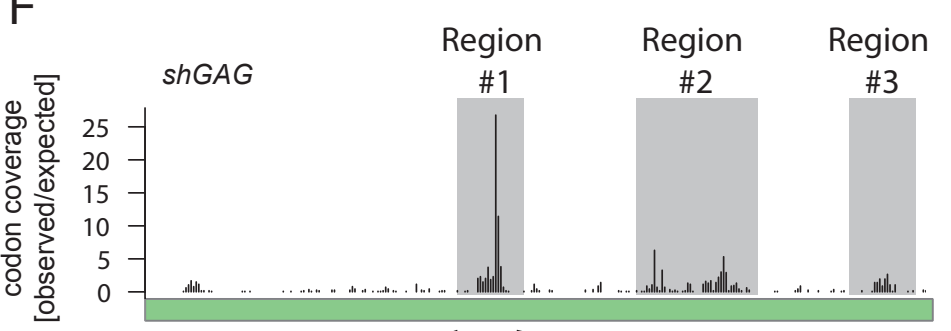

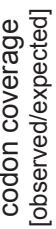

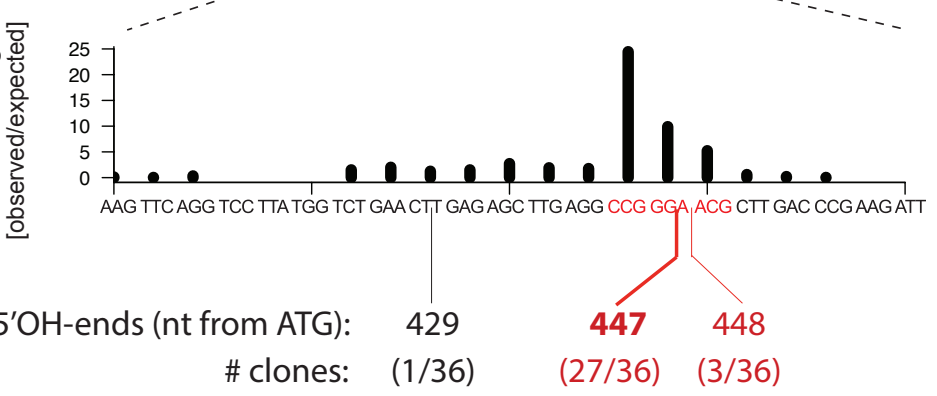

$(27 / 36)$

$(3 / 36)$

Figure 7. Intense, discrete ribosome stalling on shGAG correlates with RDR6-dependent siRNA accumulation.

(A) RIBO-seq coverage profiles from 35S:EVD in WT or rdr6. RPM: Reads per million. (B) Ribosomal footprints on shGAG in rdr6 displaying codon occupancy at P-sites to calculate codon coverage. The coverage observed at each codon position was divided by the expected mean coverage along the entire GAG coding sequence. (C) Maximal individual codon coverage over the expected coverage for all translated transcripts of Arabidopsis. Vertical lines indicate the strength of stalling sites of shGAG in the WT or rdr6 background. Percentages specify the proportion of transcripts with more pronounced stalling events than shGAG. (D) Overlay between 35S:EVD siRNAs (red) and RIBO-seq profiles (black) in the WT background. (E) Schematic representation of putative ribosome stalling-linked mRNA breakage generating $5^{\prime} \mathrm{OH}$ ends. Lack of 5'PO 4 prevents XRN 5'-> 3' exonucleolytic activity (see Fig.6A), granting the RNA to be used as template by RDR6. (F) Overlap between ribosomal footprints and mapping of $5{ }^{\prime} \mathrm{OH}$ ends from 35S:EVD in $r d r 6$ cloned through RtbC ligation. Regions investigated are highlighted in grey. $5^{\prime} \mathrm{OH}$ ends were only successfully cloned from region \#1. Alignment of sequenced clones to EVD is displayed in Fig.S9. 